NASA Contractor Report 185291

अ-

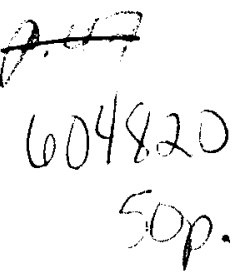

\title{
Green's Functions for Dislocations in Bonded Strips and Related Crack Problems
}

R. Ballarini and H.A. Luo

Case Western Reserve University

Cleveland, Ohio

September 1990

Prepared for

Lewis Research Center

Under Grant NAG3-856

\section{N/SA}

National Aeronautics and

Space Administration

(NASA-CR-135291) GREEN'S FUNCTIONS FOR

DISLOCATIONS IN EONDED STRIPS ANO RELATED

N90-28878

CRACK PRUgLEMS Final Report: (Case Western

Reserve Univ.) $47 \mathrm{p} \quad \mathrm{CSCL} 20 \mathrm{~K}$ 


\title{
Green's Functions for Dislocations in Bonded Strips and Related Crack Problems
}

\author{
R. Ballarini and H.A. Luo \\ Department of Civil Engineering \\ Case Western Reserve University \\ Cleveland, Ohio 44106, U.S.A.
}

\begin{abstract}
Green's functions are derived for the plane elastostatics problem of a dislocation in a bimaterial strip. Using these fundamental solutions as kernels, various problems involving cracks in a bimaterial strip are analyzed using singular integral equations. For each problem considered, stress intensity factors are calculated for several combinations of the parameters which describe loading, geometry and material mimatch.
\end{abstract}

\section{Introduction}

The mechanical behavior of bimaterial interfaces in composite materials is currently a topic of considerable interest to the applied mechanics commnnity. Many analytical, numerical and experimental investigations have been conducted recently to gain a better understanding of how these interfaces affect bulk composite properties such as strength, stiffeas and toughness. The mechanisms of cracking or debonding along bimaterial interfaces are of particular interest in brittle compoxites since it has been established experimentally and through micromechanical models that certain desired properties can only be achieved in such materials if the cracks which initiate in the matrix are deflected by the fibers along the fiber-matrix interface [1]. It is clear that micromechanical analyses will continue to play an important role in analyzing and designing brittle composites to ensure this desired failnre 
sequence. Moreover, experimental programs are necessary to measure the fracture tonghness of the fiber-matrix interface becanse the conditions required for the desired behavior involve the relative toughness between the fiber and the interface.

Mficromechanical models which involve relatively simple geometries such as cracks in infinite and semi-infinite plane bodies have been handled using distributed dislocations and singular integral equations [2]. For complicated finite geometries, on the other hand, the finite element method has gained popularity $[3,4]$. The singular integral equation method has two advantages. First, it leads to accurate results for stress intensity factors. Second, once the Green's functions are derived and the equations are set ap, parameter studies can be performed by simply varying the dimensionless parameters which describe the loading, crack length and geometry. Unfortunately, if the geometry of the problem is complicated, the method is not feasible becanse it is very difficult to derive the kernels. The real advantage of the finite element method is its ability to model complicated geometries. If proper care is taken, the method also produces accurate results. Parameter studies, on the other hand, are time consuming and relatively cumbersome.

This paper addresses a class of problems which involve cracks in bonded strips. The motivation of this work came from a desire to develop a compater program which conld be used to calculate stress intensity factors and energy release rates for bimaterial fracture specimens being developed at C.W.R.U., and model the geometry of the so-called Santa Barbara mixed-mode specimen shown in Figure 1a. The latter "T-crack" configuration was developed by Charalambides et al. [3,4] at the University of California at Santa Barbara to measure the fracture resistance of bimaterial interfaces. To interpret experimental results and to gaide the design of the specimen, they developed a finite element approach to characterize trends in stress intensity factors, energy release rates and center point displacements with specimen dimensions, elastic properties and crack length. As discussed in $[3,4]$, the stress intensity factors and energy release rate for the crack growing along the interface exhibit steady state behavior as a result of the constant moment within the inner losding points. The shortest crack for which results were calculated in $[3,4]$ is approximately $a / l=0.0938$ (or $a / h_{2}=0.3127$ ). The results showed that this crack length is already 
at steady state. One of the questions left nnanswered is: how long does the crack have to grow along the interface before it reaches steady state? To answer this question the problem is modeled in this paper using the singular integral equation technique. Besults will be presented for relatively short crack lengths as well as for the case $a=0, c \leq h_{z}$ (Fig.1c, Fig.1d, Fig.1e). It should be noted that at the end of this work the anthors learned that Charalambides has recently obtained results for both the transient region and for the three-point loading configuration.

In the next section Green's functions are derived for edge dislocations in a bimaterial strip. These fundamental solutions can be used in turn to set ap the integral equations for all the configurations shown in Figare 1. It should be noted that this class of problems has been analyzed by $L v$ [5] and $L u$ and Erdogan [6] using Fourier transforms. In [5,6] the problems were reduced to singular integral equations without the use of dislocations as fundamental solutions. Dislocation densities, however, were defined eventually to set up the integral equations. In this paper the dislocations are introduced as fundamental solutions. This will enable us, in the future, to solve problems which involve non-symmetric loading and/or inclined cracks. In the third section the dislocation solntions are used to set np the integral equations for several configurations and loadings. The last section presents numerical results and comparisons with existing solntions.

\section{Fundamental Solutions and Loading Conditions}

Assume that two dissimilar elastic stripe are bonded along the $x$ axis. The upper layer $(y>0)$ is labeled by " 1 " and the lower layer $(y<0)$ is labeled by "2". The Green's functions for an edge dislocation in the bimaterial strip are derived by superposing the solutions for (1) a dialocation near the interface of two bonded half-planes; and (2) a bimaterial strip loaded with boundary tractions which cancel ont those induced by problem (1).

The solution to problem (1) is well known. The stresses and displacements, which will be denoted by superscript " $(1)^{n}$, can be expressed conveniently in terms of Mushthelishvili's 
complex potentials as

$$
\begin{aligned}
& \left(\sigma_{v v}^{(1)}-i \sigma_{u v}^{(1)}\right)_{i}=\Phi_{i}+\overline{\Phi_{i}(z)}+z \overline{\Phi_{i}^{\prime}(z)}+\overline{\Psi_{i}(z)} \\
& \left(\sigma_{i v}^{(1)}+\sigma_{z i}^{(1)}\right)_{i}=2\left[\Phi_{i}(z)+\overline{\Phi_{i}(z)}\right] \\
& 2 \mu_{i}\left(\frac{\partial u(1)}{\partial x}+i \frac{\partial v(1)}{\partial x}\right)_{i}=\kappa_{i} \Phi_{i}(z)-\left[\overline{\Phi_{i}(z)}+z \overline{\Phi_{i}(z)}+\overline{\bar{Y}_{i}(z)}\right]
\end{aligned}
$$

in which the subscript $i(i=1,2)$ denotes "in region $i$; $\Phi_{1}$ and $\Psi_{1}$ correspond to the potentials for the npper half plane and $\Phi_{2}$ and $\Psi_{2}$ correspond to the potentials for the lower half plane. Moreover, $z$ is the complex variable $x+i y$, the prime denotes differentiation with respect to $z$, an overbar denotes conjugation, $\mu$ is the shear modulus, and $\alpha$ is defined in terms of Poiseon's ratio $\nu$ as $\kappa=3-4 \nu$ for plane strain, and $\kappa=(3-\nu) /(1+\nu)$ for plane stress. The complex potentials for a dislocation located at $z_{0}=x_{0}+i y_{0}$ are given by [7]

$$
\Phi_{i}=\Phi_{i}^{D}+\Phi_{i}^{C}, \quad \boldsymbol{\Psi}_{i}=\boldsymbol{\Psi}_{i}^{D}+\boldsymbol{\Psi}_{i}^{C}
$$

with

$$
\begin{aligned}
& \Phi_{1}^{D}=\Psi_{1}^{D}=0, \quad \Phi_{1}^{C}=\frac{1+\alpha}{1-\beta} \frac{A}{z-z_{0}}, \\
& \Psi_{1}^{C}=-\Phi_{1}^{C}-z \Phi_{1}^{C^{\prime}}+\frac{1+\alpha}{1+\beta}\left[\frac{\bar{A}}{z-z_{0}}+A \frac{\bar{z}_{0}-z_{0}}{\left(z-z_{0}\right)^{2}}\right] \\
& \Phi_{2}^{D}=\frac{A}{z-z_{0}}, \quad \quad \quad_{2}^{D}=A \frac{\overline{z_{0}}}{\left(z-z_{0}\right)^{2}}+\frac{\bar{A}}{z-z_{0}}, \\
& \Phi_{2}^{C}=\frac{\alpha-\beta}{1+\beta}\left[\frac{A}{z-\bar{z}_{0}}+\bar{A} \frac{\left(z-\bar{z}_{0}\right)}{\left(z-\bar{z}_{0}\right)^{2}}\right]
\end{aligned}
$$




$$
\Phi_{2}^{C}=-\Phi_{2}^{C}-z \Phi_{2}^{C^{\prime}}+\frac{\alpha+\beta}{1-\beta} \frac{\bar{A}}{z-\bar{z}_{0}}+\frac{\bar{A}}{z-z_{0}}+A \frac{\left(\bar{z}_{0}-z_{0}\right)}{\left(z-z_{0}\right)^{2}}
$$

In (3) the Dundurs constants $\alpha$ and $\beta$ are defined by

$$
\begin{aligned}
& \alpha=\frac{\mu_{1}\left(\kappa_{2}+1\right)-\mu_{2}\left(\kappa_{1}+1\right)}{\mu_{1}\left(\kappa_{2}+1\right)+\mu_{2}\left(\kappa_{1}+1\right)}, \\
& \beta=\frac{\mu_{1}\left(\kappa_{2}-1\right)-\mu_{2}\left(\kappa_{1}-1\right)}{\mu_{1}\left(\kappa_{2}+1\right)+\mu_{2}\left(\kappa_{1}+1\right)},
\end{aligned}
$$

and

$$
A=\frac{C}{2 \pi i} \frac{\left(1-\beta^{2}\right)}{(1-\alpha)} b, \quad C=\frac{2 \mu_{1}(1-\alpha)}{\left(\kappa_{1}+1\right)\left(1-\beta^{2}\right)}
$$

where $b=b_{z}+i b_{y}$ with $b_{z}$ and $b_{y}$ being the $x$ and $y$ components of the Burgers vector. We next compnte the stresses due to a dislocation $b_{1}=b_{1 x}+i b_{1 y}$ at the interface $\left(z_{0} \rightarrow 0\right)$ and due to a disiocation $b_{2}=b_{2 x}$ located at $x=0, y=\zeta<0$. The first dislocation corresponds to the Green's function. for the interface crack, while the second corresponds to the fondamental solution for the vertical crack. For $z_{0} \rightarrow 0$, the stresses become

$$
\sigma_{x y}^{(1)}+i \sigma_{w}^{(1)}=\left[\frac{C}{\pi} \frac{1}{x}-i \beta C \delta(x)\right] b_{1}
$$

for $y=0$,

$$
\begin{aligned}
\sigma_{w \theta}^{(1)}+i \sigma_{w}^{(1)} & =\frac{C}{\pi}\left(\frac{x}{r^{2}}-i \beta \frac{y}{r^{2}}\right) b_{1}-\frac{C}{\pi}(1+\beta)\left[\frac{2 x y^{2}}{\mu^{4}}\right. \\
& \left.+i\left(\frac{2 y^{3}}{r^{4}}-\frac{y}{r^{2}}\right)\right] \bar{b}_{1}
\end{aligned}
$$


for $y>0$, and

$$
\begin{aligned}
& \sigma_{i y}^{(1)}+i \sigma_{y y}^{(1)}=\frac{C}{\pi}\left(\frac{x}{r^{2}}+i \beta \frac{y}{r^{2}}\right) b_{1}-\frac{C}{\pi}(1-\beta)\left[\frac{2 x y^{2}}{r^{4}}\right. \\
& \left.+i\left(\frac{2 y^{3}}{r^{4}}-\frac{y}{r^{2}}\right)\right] b_{1}, \\
& \sigma_{2 x}^{(1)}=\frac{C}{\pi} \Re\left[D(y, x) b_{1}\right]
\end{aligned}
$$

with

$$
D(y, x)=(1-\beta) \frac{2 y^{3}}{r^{4}}-(3-2 \beta) \frac{y}{r^{2}}-i\left[(1-2 \beta) \frac{x}{r^{2}}-(1-\beta) \frac{2 x y^{2}}{r^{4}}\right]
$$

for $y<0$, where $\delta(x)$ is the Dirac delta function. Here, some care must be taken during performing the limit [8]. Meanwhile, for dislocation $b_{3}$, it can be shown that

$$
\begin{aligned}
\sigma_{2 y}^{(1)}+i \sigma_{w y}^{(1)} & =-\frac{C}{\pi}\left[\frac{2 x(y-\zeta)^{2}}{r_{1}^{4}}-\frac{x}{r_{1}^{2}}+\beta \frac{2 x(y-\zeta)^{2}}{r_{1}^{4}}\right. \\
& \left.+4 \beta \zeta \frac{x(y-\zeta)}{r_{1}^{4}}\right] b_{2}-i \frac{C}{\pi}\left[\frac{2(y-\zeta)^{3}}{r_{1}^{4}}-\frac{y-\zeta}{r_{1}^{2}}\right. \\
& \left.+2 \beta \frac{(y-\zeta)^{3}}{r_{1}^{4}}+4 \beta \zeta \frac{(y-\zeta)^{2}}{r_{1}^{4}}-2 \beta \zeta \frac{1}{r_{1}^{2}}\right] b_{2}
\end{aligned}
$$

for $y \geq 0$,

$$
\begin{aligned}
\sigma_{x y}^{(1)}+i \sigma_{y y}^{(1)} & =-\frac{C}{\pi(1+\alpha)}\left\{\left(1-\beta^{2}\right)\left(\frac{2(y-\zeta)^{2} x}{r_{1}^{4}}-\frac{x}{r_{1}^{2}}\right)+\left(\alpha+\beta^{2}\right)\left(\frac{2(\zeta+y)^{2} x}{r_{1}^{4}}\right.\right. \\
& \left.-\frac{x}{r_{2}^{2}}\right)-(1+\alpha) \beta \frac{2(\zeta+y)^{2} x}{r_{2}^{4}}+(\alpha-\beta)(1-\beta) \zeta\left(\frac{4(\zeta+y) x}{r_{2}^{4}}\right.
\end{aligned}
$$




$$
\begin{aligned}
& \left.\left.-\frac{16(\zeta+y)^{3} x}{r_{2}^{6}}\right)+2(\alpha-\beta)(1-\beta) \zeta^{2}\left(\frac{8(\zeta+y)^{2} x}{r_{2}^{6}}-\frac{2 x}{r_{2}^{4}}\right)\right\} b_{2}, \\
& +i \frac{C}{\pi(1+\alpha)}\left[\left(1-\beta^{2}\right)\left(\frac{y-\zeta}{r_{1}^{2}}-\frac{2(y-\zeta)^{3}}{r_{1}^{4}}\right)-\left(\alpha+\beta^{2}\right)\right. \\
& \times\left(\frac{2(\zeta+y)^{3}}{r_{2}^{4}}-\frac{\zeta+y}{r_{2}^{\frac{2}{2}}}\right)+(1+\alpha) \beta \frac{2(\zeta+y)^{3}}{r_{2}^{4}} \\
& \left.-2(\alpha-\beta)(1-\beta) \zeta \frac{1}{r_{2}^{\frac{2}{2}}}\right)-(\alpha-\beta)(1-\beta) \zeta\left(\frac{8(\zeta+y)^{2}}{r_{2}^{4}}\right. \\
& \left.\left.-\frac{16(\zeta+y)^{4}}{r_{2}^{6}}\right)+2(\alpha-\beta)(1-\beta) \zeta^{2}\left(\frac{6(\zeta+y)}{r_{2}^{4}}-\frac{8(\zeta+y)^{3}}{r_{2}^{6}}\right)\right] b_{2}
\end{aligned}
$$

for $y \leq 0$, where

$$
r_{1}^{2}=x^{2}+(y-\zeta)^{2}, \quad r_{2}^{2}=x^{2}+(y+\zeta)^{2} .
$$

In particular, when $y=0$

$$
\sigma_{w 1}^{(1)}(x, 0)+i \sigma_{w}^{(1)}(x, 0)=\frac{C}{\pi} E(x, \zeta) b_{2}
$$

with

$$
E(x, \zeta)=\frac{x+i \zeta}{r_{*}^{2}}-2(1-\beta) x \zeta \frac{\zeta+i x}{r_{*}^{4}}
$$

where $r_{*}^{2}=x^{2}+\zeta^{2} ;$ and when $y<0$

$$
\sigma_{z=}^{(1)}(0, y)=-\frac{C\left(1-\beta^{2}\right)}{x(1+\alpha)}\left[\frac{1}{y-\zeta}+F(y, \zeta)\right] b_{2}
$$

(15)

7 
where

$$
\begin{gathered}
F(y, \zeta)=\frac{\left(\alpha+\beta^{2}\right)}{\left(1-\beta^{2}\right)} \frac{1}{y+\zeta}+\frac{2(\alpha-\beta)}{1+\beta} \frac{\zeta}{(y+\zeta)^{2}} \\
-\frac{4(\alpha-\beta)}{1+\beta} \frac{\zeta^{2}}{(y+\zeta)^{3}}
\end{gathered}
$$

The solution to problem (2) is obtained by using Fourier transformation techniques. Withont loes of generality, consider a bimaterial strip loaded with surface tractions which are symmetric about the $y$-axis. The Fourier transform of displacement is as follows:

$$
\begin{aligned}
& U_{i}(\xi, y)=\int_{0}^{\infty} u_{i}(x, y) \sin \xi x d x \\
& V_{i}(\xi, y)=\int_{0}^{\infty} v_{i}(x, y) \cos \xi x d x
\end{aligned}
$$

The general solntions for $V_{i}$ and $V_{i}$ are [9]:

$$
\begin{aligned}
U_{i}(\xi, y) & =\left(A_{i 1} h_{2}+A_{i 2} y\right) e^{-\xi y}+\left(A_{i 3} h_{2}+A_{i 1} y\right) e^{\xi y} \\
V_{i}(\xi, y) & =\left[A_{i 1} h_{2}+\left(\alpha_{i} / \xi+y\right) A_{i 2}\right] e^{-\xi y}+1-A_{i j} h_{2} \\
& \left.+\left(\kappa_{i} / \xi-y\right) A_{i 4}\right] e^{\xi y}
\end{aligned}
$$

with the unknown coefficients determined by continuity and boundary conditions along the interface: 


$$
\begin{aligned}
& A_{11}+A_{13}-A_{21}-A_{23}=0 \\
& A_{11}+\frac{\kappa_{1}}{\xi h_{2}} A_{12}-A_{1 s}+\frac{\kappa_{1}}{\xi h_{2}} A_{14}-A_{21}-\frac{\kappa_{2}}{\xi h_{2}} A_{22} \\
& +A_{2 s}-\frac{m_{2}}{\xi h_{2}} A_{24}=0 \\
& \xi h_{2} A_{11}+2\left(1-\nu_{1}\right) A_{12}+\xi h_{2} A_{13}-2\left(1-\nu_{1}\right) A_{14}-\frac{\mu_{2}}{\mu_{1}} \\
& \times\left[\xi h_{2} A_{21}+2\left(1-\nu_{2}\right) A_{22}+\xi h_{2} A_{2 s}-2\left(1-\nu_{2}\right) A_{24}\right]=0 \\
& \xi h_{2} A_{11}+\left(1-2 \nu_{1}\right) A_{12}-\xi h_{2} A_{19}+\left(1-2 \nu_{1}\right) A_{14}-\frac{\mu_{2}}{\mu_{1}} \\
& \times\left[\xi h_{2} A_{21}+\left(1-2 w_{2}\right) A_{22}-\xi h_{2} A_{23}+\left(1-2 \nu_{2}\right) A_{24}\right]=0 \\
& -\left[\xi\left(A_{11} h_{2}+A_{12} h_{1}\right)+2\left(1-\nu_{1}\right) A_{12}\right] e^{-\xi h_{1}}+\left[-\xi\left(A_{13} h_{2}\right.\right. \\
& \left.\left.+A_{14} h_{1}\right)+2\left(1-\nu_{1}\right) A_{14}\right] e^{\xi h_{1}}=f_{1}(\xi) \\
& -\left[\xi\left(A_{11} h_{2}+A_{12} h_{1}\right)+\left(1-2 \nu_{1}\right) A_{12}\right] e^{-\xi h_{1}}+\left[\xi\left(A_{15} h_{2}+A_{14} h_{1}\right)\right. \\
& \left.-\left(1-2 \nu_{1}\right) A_{14}\right] e^{\xi h_{1}}=f_{2}(\xi) \\
& -\left[\xi h_{2}\left(A_{21}-A_{22}\right)+2\left(1-\nu_{2}\right) A_{22}\right] e^{\xi h_{2}}+\left[-\xi h_{2}\left(A_{25}-A_{24}\right)\right. \\
& \left.+2\left(1-\nu_{2}\right) A_{24}\right] e^{-f h_{2}}=f_{3}(\xi)
\end{aligned}
$$




$$
\begin{gathered}
-\left[\xi h_{2}\left(A_{21}-A_{22}\right)+\left(1-2 \nu_{2}\right) A_{22}\right] e^{\xi h_{2}}+\left[\xi h_{2}\left(A_{23}-A_{24}\right)\right. \\
\left.-\left(1-2 \nu_{2}\right) A_{24}\right] e^{-\xi h_{2}}=f_{4}(\xi)
\end{gathered}
$$

where the first four equations represent the continnity of $u, v, \sigma_{w y}$ and $\sigma_{z y}$ along the interface, respectively; the last four equations specify the boundary conditions at $y=h_{1}$ and $y=-h_{2}$. The functions $f_{1}(\xi)$ and $f_{2}(\xi)$ are related to the Fourier transforms of the loadings on the top surface of the upper strip, while $f_{3}(\xi)$ and $f_{4}(\xi)$ are related to the Fonrier transforms of the loadings on the bottom surface of the lower strip. To eliminate the nnwanted surface traction along the boundaries due to each dislocation, for example $b_{x}$, in problem (1), functions $f_{1}(\xi), f_{2}(\xi), f_{3}(\xi)$ and $f_{4}(\xi)$ are set to be

$$
\begin{aligned}
& f_{1}(\xi)=-\frac{1}{2 \mu_{1}} \int_{0}^{\infty} \sigma_{w y}^{(1)}\left(x, h_{1}\right) \cos \xi x d x \\
& f_{2}(\xi)=-\frac{1}{2 \mu_{1}} \int_{0}^{\infty} \sigma_{w y}^{(1)}\left(x, h_{1}\right) \sin \xi x d x \\
& f_{3}(\xi)=-\frac{1}{2 \mu_{2}} \int_{0}^{\infty} \sigma_{w y}^{(1)}\left(x,-h_{2}\right) \cos \xi x d x \\
& f_{4}(\xi)=-\frac{1}{2 \mu_{2}} \int_{0}^{\infty} \sigma_{x y}^{(1)}\left(x,-h_{2}\right) \sin \xi x d x .
\end{aligned}
$$

It can be shown that equations (19) are also applicable to the anti-symmetric problem. The $f_{i}(\xi)$ 's have been evaluated for each case and are given in the Appendix.

After the $A_{i j}$ 's are solved, the stress components due to problem (2), which are denoted by soperscripts "(2)", are readily abtained by inverse Fourier transformation. For the 
symmetric problem, e. g. for dislocation $b_{3}$,

$$
\begin{aligned}
\sigma_{z y}^{(2)}(x, 0)= & \frac{4 \mu_{1} b_{2}}{\pi} \int_{0}^{\infty}\left[\xi h_{2}\left(A_{13}-A_{11}\right)-\left(1-2 \nu_{1}\right)\left(A_{12}+A_{14}\right)\right] \\
& \times \sin \xi x d \xi \\
\sigma_{w}^{(2)}(x, 0)= & \frac{4 \mu_{1} b_{2}}{\pi} \int_{0}^{\infty}\left[-\xi h_{2}\left(A_{11}+A_{13}\right)+2\left(1-\nu_{1}\right)\left(A_{14}-A_{12}\right)\right] \\
& \times \cos \xi x d \xi
\end{aligned}
$$

and

$$
\begin{gathered}
\sigma_{x=}^{(2)}(x, y)=\frac{4 \mu_{2} b_{x}}{\pi} \int_{0}^{\infty}\left\{\left[\xi\left(A_{21} h_{2}+A_{20 y}\right)-2 \nu_{2} A_{22}\right] e^{-\xi y}\right. \\
\left.+\left[\xi\left(A_{23} h_{2}+A_{24} y\right)+2 \nu_{2} A_{24}\right] e^{\xi y}\right\} \cos \xi x d \xi
\end{gathered}
$$

where $y<0$. For the anti-oymmetric problem, e. g. for dislocation $b_{y}$, the above relationship is still valid if $\sin \xi x$ is replaced by $\cos \xi x$ and $\cos \xi x$ is replaced by $-\sin \xi x$.

For convenience, in subsequent discussions introduce the following definitions for the streases produced by problem (2):

$$
\begin{aligned}
& \sigma_{3 y}^{(z)}(x, 0)+i \sigma_{y y}^{(2)}(x, 0) \equiv G_{1}(x) b_{1 s}+G_{2}(x) b_{1 y}+G_{3}(x ; \zeta) b_{2 x} \\
& \sigma_{m s}^{(z)}(x, y) \equiv H_{1}(y ; x) b_{1 x}+H_{2}(y ; x) b_{1 y}+H_{3}(y ; \zeta, x) b_{2 x} .
\end{aligned}
$$


We wish to point out that the fundamental solutions derived above can be used to solve a large class of problems. The purpose of this paper is to present the methodology. Thus we will consider only three types of loadings. These include three and four point bending (Fig.1a), constant pressure along the crack surfaces, and a temperature change of the composite strip (Fig.1d). Consider first a perfectly bonded bimaterial beam subjected to fourpoint bending. The stresses in the strip dne to this loading condition can be calculated using composite beam theory if the strip is long enongh. However, since we may be interested, in the future, in analyzing relatively short beams, an elasticity solntion is obtained in the same manner as for problem (2) of the dislocation solution. The applied loads are represented by Dirac delta functions at the load points. That is $\sigma_{y w}\left(x, h_{1}\right)=-p[\delta(x-d)+\delta(x+d)] / 2$ and $\sigma_{9 y}\left(x,-h_{2}\right)=-p[\delta(x-e)+\delta(x+e)] / 2$. The fonctions $f_{1}, f_{2}, f_{3}$ and $f_{4}$ for this case are listed in the Appendix. To evaluate the stresses which arise from this loading, it may seem natnral to proceed in the same exact manner as for the dislocation solntions. However, tremendous care must be taken when evaluating the stresses along the interface and along the line $x=0$ due to this loading condition, because the integrals in the inverse transformations converge very slowly. To improve the convergence the integrands are first evaluated at $\xi=\infty$. The dominant portion of these limits, which correspond to half-space solutions, are subtracted from and added to the integrands, and the added integrals are evaluated in closed form. The same procedure is also applied to the dislocation $b_{2}$ when it is cloee to the bottom surface. For brevity the details of the procedure are not given here, but can be recovered in similar analyses presented in $[10,11]$.

Denote the stresses produced by the four point bending as

$$
\begin{aligned}
& \sigma_{z y}(x, 0)+i \sigma_{z y}(x, 0) \equiv p Q(x) / h_{2} \\
& \sigma_{z x}(0, y) \equiv p R(y) / h_{2}
\end{aligned}
$$

respectively, where $p$ is the force per unit thickmess. These will be used in section 3 to set 
up the integral equations.

The next loading condition consiats of a uniform change of temperature $\Delta T$ of the composite strip. The thermal stresses in the strip are calculated nsing beam theory [12]. In particular, the stress in layer 2 along the line $x=0$ is given in terms of the thermal expansion coefficients $\alpha_{1}$ and $\alpha_{2}$ by

$$
\sigma_{a z}=-\left(\alpha_{2}-\alpha_{1}\right) \Delta T E_{2}^{*}\left(1+\frac{3}{2} \frac{y}{h_{2}}\right) /\left(1+\frac{E_{2}^{*} h_{2}}{E_{1}^{*} h_{1}}\right)
$$

with

$$
E^{*}=\frac{E}{1-\nu^{2}}
$$

\section{Integral Equations}

Several problem configurations are depicted in Figure 1. The coordinate system is chosen such that the $x$ axis lies along the interface of the bimaterial strip, and the $y$ axis along the vertical crack so that layer 1 is at $y>0$ and layer 2 is at $y<0$. The integral equations for the four point bending problem shown in Fig.1a are set up first. The stresses along the lines $y=0$ and $x=0(y \leq 0)$ are given by the summation of contributions from problem (1), problem (2) and the four point bend load (24). Replacing the dislocations by a distribution of dislocations enables us to satisfy the traction boundary conditions along the crack. This procedure leads to the following set of conpled singular integral equations:

$$
\begin{aligned}
& \int_{-1}^{1} \frac{B_{1}\left(t_{0}\right)}{t-t_{0}} d t_{0}-i \beta \pi B_{1}(t)+\int_{-1}^{1} K_{1}\left(t, t_{0}\right) B_{1}\left(t_{0}\right) d t_{0} \\
& \quad+\int_{-1}^{1} K_{2}\left(t, t_{0}\right) \bar{B}_{1}\left(t_{0}\right) d t_{0}+\int_{-1}^{1} K_{5}\left(t, t_{0}\right) B_{2}\left(t_{0}\right) d t_{0}=p_{1}(t) \\
& \int_{-1}^{1} \frac{B_{2}\left(t_{0}\right)}{t-t_{0}} d t_{0}+\int_{-1}^{1} K_{4}\left(t, t_{0}\right) B_{2}\left(t_{0}\right) d t_{0}+\int_{-1}^{1} K_{5}\left(t, t_{0}\right) B_{1}\left(t_{0}\right) d t_{0}
\end{aligned}
$$




$$
+\int_{-1}^{1} K_{6}\left(t, t_{0}\right) \bar{B}_{1}\left(t_{0}\right) d t_{0}=p_{2}(t)
$$

together with the conjugate of the first equation in (26) where the dislocation densities are given by $B_{1}(t)=B_{1 x}(a t)+i B_{1 y}(a t)$ and $B_{2}(t)=B_{2 \varepsilon}\left(h_{2}(t-1) / 2\right)$ with

$$
\begin{aligned}
& B_{1 \varepsilon}(x)=-\frac{C h_{2}}{p} \frac{\partial}{\partial x}\left[u\left(x, 0^{+}\right)-u\left(x, 0^{-}\right)\right] \\
& B_{1 y}(x)=-\frac{C h_{2}}{p} \frac{\partial}{\partial x}\left[v\left(x, 0^{+}\right)-v\left(x, 0^{-}\right)\right] \\
& B_{2 \varepsilon}(y)=-\frac{C h_{2}}{p} \frac{\partial}{\partial y}\left[u\left(0^{+}, y\right)-u\left(0^{-}, y\right)\right]
\end{aligned}
$$

and

$$
\begin{aligned}
& K_{1}\left(t, t_{0}\right)=\frac{a \pi}{2 C}\left[G_{1}\left(a\left(t-t_{0}\right)\right)-i G_{2}\left(a\left(t-t_{0}\right)\right)\right] \\
& K_{2}\left(t, t_{0}\right)=\frac{a \pi}{2 C}\left[G_{1}\left(a\left(t-t_{0}\right)\right)+i G_{2}\left(a\left(t-t_{0}\right)\right)\right] \\
& K_{9}\left(t, t_{0}\right)=\frac{h_{2}}{2}\left[E\left(a t, \frac{h_{2}\left(t_{0}-1\right)}{2}\right)+\frac{\pi}{C} G_{3}\left(a t ; \frac{h_{2}\left(t_{0}-1\right)}{2}\right)\right] \\
& K_{4}\left(t, t_{0}\right)=\frac{h_{2}}{2}\left[F\left(\frac{h_{2}(t-1)}{2}, \frac{h_{2}\left(t_{0}-1\right)}{2}\right)-\frac{\pi(1+\alpha)}{C\left(1-\beta^{2}\right)}\right. \\
& \left.\quad x B_{3}\left(\frac{h_{2}(t-1)}{2} ; \frac{h_{2}\left(t_{0}-1\right)}{2}, 0\right)\right] \\
& K_{5}\left(t, t_{0}\right)=-\frac{a(1+\alpha)}{2\left(1-\beta^{2}\right)}\left\{D\left(y,-x_{0}\right)+\frac{\pi}{C}\left[H_{1}\left(y ;-x_{0}\right)-i H_{2}\left(y ;-x_{0}\right)\right]\right\} \\
& K_{6}\left(t, t_{0}\right)=\frac{K_{5}\left(t, t_{0}\right)}{}
\end{aligned}
$$




$$
\begin{aligned}
& p_{1}(t)=-\pi Q(a t) \\
& p_{2}(t)=\frac{1+\alpha}{1-\beta^{2}} \pi R\left(\frac{h_{2}(t-1)}{2}\right) .
\end{aligned}
$$

If the vertical crack is not present, $B_{2}$ is set equal zero and only the first equation of (26) is enforced. If the interface crack is not present, on the other hand, the first of equations (26) is not enforced and $B_{1}$ is set equal to zero in the second equation. For the edge crack whose length $c<h_{2}$, the dislocation density is instead represented as $B_{2}(t)=B_{22}(c(t-1) / 2)$, and in the expression of the kernel $K_{1}$ given by (28) $h_{2}$ needs to be replaced by c. Other loading conditions can be treated by rnodifying functions $p_{1}$ and $p_{2}$.

The integral equations can be solved numerically by representing esch dislocation density in terms of a regular function and a characteriatic function with the proper singularities at the end points. Thus, let

$$
B_{1}(t)=\frac{\phi_{1}(t)}{(1-t)^{\gamma}(1+t)^{1-\gamma}}, \quad B_{2}(t)=\frac{\phi_{2}(t)}{(1-t)^{\lambda} \sqrt{1+t}}
$$

where $\phi_{1}(t)$ and $\phi_{2}(t)$ are regular continuous functions which are approximated as piecewise quadratic [13], and

$$
y=\frac{1}{2}+i \epsilon, \quad \epsilon=\frac{1}{2 \pi} \log \frac{1-\beta}{1+\beta}
$$

The exponent $\lambda$ is taken as 0.5 for the T-crack (Fig.1a) and for an edge crack whose tip does not touch the interface (Fig.1c, Fig.1d). If the tip of the edge crack tonches the interface, $\lambda$ is determined by the following characteriatic equation [14,15]: 


$$
\cos \pi \lambda-\frac{2(\beta-\alpha)}{1+\beta}(1-\lambda)^{2}-\frac{\alpha+\beta^{2}}{1-\beta^{2}}=0
$$

Using the method developed by Miller and Keer [13], integral equations (26) can be reduced to a set of algebraic equations.

The additional conditions to be satisfied are as follows. For the $T$-crack

$$
\begin{aligned}
& \int_{-1}^{1} B_{1 y}(t) d t=0 \\
& \phi_{2}( \pm 1)=0 \\
& \int_{-1}^{1} B_{1 x}(t) d t \neq 0 .
\end{aligned}
$$

Equation (32) is the closure condition for the horizontal crack, (33) represents the condition that the stress singularities at the tips of the vertical notch are less than square root, and (34) implies the blontness at $x=0$. As will be discussed in the next section, the representation of the dislocation density for the T-crack is not rigorons. However, the error introduced is acceptable for the configurations considered in this paper.

For a singie interface crack

$$
\int_{-1}^{1} B_{1}(t) d t=0
$$

and for a single edge crack

$$
\phi_{2}(-1)=0
$$




$$
\int_{-1}^{1} B_{2 x}(t) d t \neq 0
$$

\section{Numerical Results and Stress Intensity Factor Analysis}

The stress intensity factor $K$ of the interface crack (Fig-1b) and the T-crack (Fig.1a) are defined by [16]

$$
K=\lim _{x \rightarrow a}\left\{\sqrt{2 \pi(x-a)}(x-a)^{-i c}\left[\sigma_{w}(x, 0)+i \sigma_{z y}(x, 0)\right]\right\}
$$

It can be shown that in terms of $\phi_{1}(t)$, which is determined by equation (26) with (28) and (29), this complex stress intensity factor can be expressed as

$$
K=\frac{i p}{h_{2}} \sqrt{\pi a\left(1-\beta^{2}\right)}(2 a)^{-i \epsilon} \overline{\phi_{1}(1)}
$$

for the four point bending load. For: an edge crack whose tip does not touch the interface

$$
K=\lim _{y \rightarrow\left(c-h_{z}\right)}\left[\sqrt{2 \pi\left(h_{2}+y-c\right)} \sigma_{z=z}(0, y)\right],
$$

and similarly in terms of $\phi_{2}(t)$

$$
K=\frac{p}{h_{2}} \frac{\left(1-\beta^{2}\right)}{(1+\alpha)} \sqrt{\pi c / 2} \phi_{2}(1)
$$

for the four point bending. For an edge crack whose tip touches the interface 


$$
K=\lim _{y \rightarrow 0}\left[\sqrt{2 \pi} y^{\lambda} \sigma_{u s}(0, y)\right]
$$

and

$$
K=\frac{p}{h_{2}} \frac{\left(1-\beta^{2}\right)}{(1+\alpha)} \sqrt{\pi}\left(h_{2} / 2\right)^{\lambda} \phi_{2}(1)
$$

For the other loading cases, the factor $p / h_{2}$ in (39) (41) and (43) is replaced correspondingly.

The energy release rate of the interface cracks in the considered plane strain problems is given by [17]

$$
G=\left[\left(1-\nu_{1}\right) / \mu_{1}+\left(1-\nu_{2}\right) / \mu_{2}\right] K \bar{K} / 4 \cosh ^{2}(\pi \epsilon)
$$

In order to check the lengthy algebra and the numerical scheme, two configarations which have previonsly been analyzed are considered first: a pressurized crack between two bonded dissimilar layers and a pressurized vertical edge crack in the bottom layer of a composite beam. Fig.2 shows the dimensionless stress intensity factors $\dot{K} / \sigma_{0} \sqrt{a}=$ $\lim _{w \rightarrow a}\left[(a-x)^{1-\gamma}(a+x)^{\gamma}\left(\sigma_{y v}+i \sigma_{z y}\right)\right] / \sigma_{0} a$ versus $0.1 \leq h_{1} / 2 a \leq 4$ for the interface crack with $h_{2} / h_{1}=3, \nu_{1}=\nu_{2}=0.3, \mu_{1} / \mu_{2}=3$ and $\mu_{1} / \mu_{2}=10$, respectively. The results are plotted in terms of this definition of stress intensity factor for comparison with reference [18]. For $h_{1} / 2 a>0.5$ the results agree with those presented in [18] (no results were given there for $\left.h_{1} / 2 a<0.5\right)$. As expected, when the crack becomes shorter, the stress intensity factor converges to $1+2 i e$ which is the solntion for an interface crack between two bonded half-spaces.

For the edge crack problem a homogeneous beam and four ceramic composite strips are considered. They are: strip $1 \mathrm{Ti} / \mathrm{Al} 2 \mathrm{O} 3$ with $\nu_{1}=0.322, \nu_{2}=0.207$ and $\mu_{2} / \mu_{1}=4.129$, strip $2 \mathrm{Ni} / \mathrm{MgO}_{\mathrm{g}}$ with $\nu_{1}=0.314, \nu_{2}=0.175$ and $\mu_{2} / \mu_{1}=1.588$, strip $3 \mathrm{MgO} / \mathrm{Ni}$ and strip 4 AlrOs/ $\mathrm{Ti}$ [16]. Fig.3 and Fig-4 show the dimensionless stress intensity factors as functions of crack length and relative thickness of the layers for the four point bending case considered in $[3,4]$ where $d=5 h_{2}, e=8 \frac{1}{3} h_{2}$ and $l=3 \frac{1}{3} h_{2}$. The quantity $\sigma_{\max }$ is the axial stress at the 
lower surface of the composite beam calculated using composite beam theory. It is given by

$$
\sigma_{\max }=\frac{M m H_{2}}{\left(I_{1}+m I_{2}\right)}
$$

with

$$
\begin{aligned}
& m=\frac{\mu_{2}\left(1-\nu_{1}\right)}{\mu_{1}\left(1-\nu_{2}\right)}, \quad \delta=h_{1} / h_{2}, \\
& H_{1}=h_{1}+h_{2}-H_{2}, \quad H_{2}=\frac{h_{2}}{2(m+\delta)}\left(m+2 \delta+\delta^{2}\right), \\
& I_{1}=\frac{1}{12} h_{1}^{3}+h_{1}\left(H_{1}-\frac{h_{1}}{2}\right)^{2}, \quad I_{2}=\frac{1}{12} h_{2}^{3}+h_{2}\left(H_{2}-\frac{h_{2}}{2}\right)^{2} .
\end{aligned}
$$

where $M$ is the bending moment; for the four point bending shown in Fig.1 $M=p l / 2$. It is observed that as the crack tip approsches the interface, the stress intensity factor increases drastically if the edge crack is in the stiffer layer. In the limit, the stress intensity factor approaches infinity for this case, since it can be shown that as the crack tip hits the interface the stress singularity is grester than square root. When the crack is in the softer layer, the stress intensity factor goes to zero as the tip approaches the interface, since the singularity in this case is less than square root. When the crack is very short, namely $c / h_{2} \rightarrow 0$, the dimensionless stress intensity factor approaches 1.586, which corresponds to the solution of an edge crack in a half-space under uniform tension. When the thickness of the apper layer becomes very thin, the stress intensity factors for the bimaterial strips approach those for a homogeneous beam. It is intisresting to note that for these valnes of mismatch, the dimensionless stress intensity factors are insensitive to $c / h_{2}$ for $c / h_{2}<0.5$ and insensitive to $h_{1} / h_{2}$ for $h_{1} / h_{2}>1$. Results were also obtained for three point bending. For this geometry the nondimensional results were found to be almost exactly the same as for the four point bending.

Fig. 5 shows the stress intensity factors for the edge crack whose tip touches the interface. In this case the singularity depends on the elastic mismatch of the two layers and factor $\lambda$ 
is calculated from (31). For the combination considered here $\nu_{1}=\mu_{2}=0.3, \mu_{3} / \mu_{1}=3$ and $\mu_{2} / \mu_{1}=1 / 3$, and singularities $\lambda=0.6205$ and $\lambda=0.4005$, respectively. Notice that, since the stress singularities are different, it is meaningless to directly compare the values of the dimensionless stress intensity factors given in Fig.4 and Fig.5. It should be noted that these resalts do not compare at all with those in $[5,6]$.

Suppose that a bimaterial beam is abbjected to a concentrated force $p$ along its neutral axis as shown in Fig.1f where $H_{1}$ and $H_{2}$ are given by (46). The longitudial stress across the lower layer is

$$
\sigma^{(2)}=\frac{p}{h_{2}(1+\delta \Sigma)}
$$

where $\Sigma=\mu_{1}\left(1-\nu_{2}\right) /\left[\mu_{2}\left(1-\nu_{1}\right)\right]$. If the beam is subjected to the remote bending, based on the composite beam theory, the stress on the lower layer is

$$
\sigma_{x}=\sigma_{\max } \frac{H_{2}-h_{2}-y}{H_{2}}
$$

where $\sigma_{\max }$ and $H_{2}$ are given in (45) and (46). Notice that two Dundurs parameters $\alpha$ and $\beta$ can cover the elastic moduli dependence of a two-dimensional bimaterial system [19]. In Table 1 and Table 2 the stress intensity factors are given in terms of $\alpha$ and $\beta$ for an edge crack with $c / h_{2}=0.5$ under the above two loading systems. Table 3 shows the singularity $\lambda$ of the edge crack whose tip touches the interface. The corresponding stress intensity factors are given for this class of edge cracks.

Fig. 6 and Fig. 7 show the stress intensity factors of the edge craclos for the thermal loading case. The sign of the stress intensity factor changes when the crack length is approximately equal to $0.6 h_{2}$ as a result of bending. If additional loads are superimposed, these results suggest that wack growth can either be accelerated when $\left(\alpha_{2}-\alpha_{1}\right) \Delta T<0$ or slowed down when $\left(\alpha_{2}-\alpha_{1}\right) \Delta T>0$ by the introduction of a temperature change. 
The last example considers the T-crack, which is referred to as the Santa Barbara opecimen, shown in Figare la where $d=5 h_{2}, e=8 \frac{1}{3} h_{2}$ and $l=3 \frac{1}{3} h_{2}$. Resnlts were calculated for crack lengths which are shorter than thoee presented in $[3,4]$. Figure 8-14 show the dimensionless stress intensity factors $K h^{i c} h^{3 / 2} / p l$, energy release rates $G$ and the phase angles, defined as the argument of $K h^{\text {ie }}$, for strips with $\nu_{1}=\nu_{2}=0.3$ and $\mu_{1} / \mu_{2}=1, \mu_{1} / \mu_{2}=2.5$, $\mu_{1} / \mu_{2}=5, \mu_{1} / \mu_{2}=10$, respectively. The results obtained in $[3,4]$ for $a / h_{2}>0.5$ are superposed on the figures. The agreement is observed to be quite good. It is obeerved that steady state begins at crack lengths equal to $h_{2}$. Results for $a / h_{2}$ less than 0.1 were not calculated becanse we believe that accurate solutions cannot be obtained for these cases using the present formulation, since the integral equations are conpled. To obtain accurate results for smail value of $a / h_{2}$ one would need to formulate the problem in terms of a single integral equation whose kemel incledes an analytic solution for the interaction of the two cracks.

It should be mentioned that for crack lengths greater than $1.5 h_{2}$ convergent results were not obtained as a result of nsing a distribution of dislocations density which is continnous at $x=0, y=0$. Because the vertical crack intersects the interface crack, the slope of the horizontal crack is discontinnous at $(0,0)$. The numerical scheme implicitly assumes that the slope there is zero. We believe it is this error which leads to poor results for long crack lengths, for which the slope at $(0,0)$ is definitely not zero. This problem is being addressed by the anthors at the present time by introducing a discontinuous distribution of dislocations.

\section{Conclusions}

An analytical formulation has been presented which can be used to solve a class of plane elastostatic problems involving cracted bimaterial beams. Results were calculated only for a few geometric configarations and gymmetric loadings. However, since the analysis relies on fundamental solutions for dislocations in bonded strips, other configurations such as inclined or curved cracks and/or non-symmetric loadings can be treated with minor modifications. 
For the T-crack, when the interface crack is relatively short, the results obtained using the singular integral equation approsech compare very well with those obtained in $[3,4]$ using the finite element method. However, for such configurations, which involve discontinuous dislocation densities at the intersection of the two cracks, poor resnlts were obtained for relatively long interface cracts. This problem can be treated by modifying the numerical scheme to handle discontinuous dislocation densities.

\section{Acknowledgement}

Financial support was provided by the Defense Advanced Besearch Projects Agency through the University Research Initiative Program of C.W.R.U. nnder ONR Contract N-0013-86-K-0773 and by Lewis Research Center ander Grant NAG3-856. The computations were performed on a Cray which was made available through a grant from the Ohio Snpercompater Center. 


\section{References}

1 A.G. Evans and D.B. Marshall, Acta Metallurgica 37 (1989) 2567-2583.

2 M.-Y. He and J.W. Huchinson, International Journal of Solids \& Structures 25 (1989) $1053-1067$.

3 P.G. Charalambides, J. Lund, A.G. Evans and R.M. MeMeeking, ASME Journal of Applied Mechanics 56 (1989) 77-82.

4 P.P.L. Matos, R.M. McMeeking, P.G. Charalambides and M.D. Drory, International Journal of Practure 40 (1989) 235-254.

3 M.-C. La, Ph.D. Dissertation, Lehigh University (1978).

6 M.-C. La and F. Erdogan, Engineering Fracture Mechanics 18 (1983) Part I, 491-506; Part II, 507-528.

7 R. Ballarini, D.J. Mukai and G.R. Miller, NASA Contractor Report 182273 (1989), also to appear in ASME Journal of Applied Mechanics.

8 M. Comninou, Philosophical Magazine 36 (1977) 1281-1283.

9 I.N. Sneddon, Fourier Transforms, McGraw Hill, New York (1951).

10 R. Ballarini, S.P. Shah and L.M.Keer, Engineering Fracture Mechanics 20 (1984) 433445.

11 P.D. Copp, Ph.D. Dissertation, Northwestern University (1986).

12 B.A. Boley and J.H. Weiner, Theory of Thermal Stresses, John Wiley \& Sons (1960).

13 G.R. Miller and L.M. Keer, Quarterly of Applied Mathematics 42 (1985) 453-465.

14 T.S. Cook and F. Erdogan, International Journal of Engineering Science 10 (1972) $677-697$. 
15 F. Erdogan and V. Biricilogln, Intemational Joumal of Engineering Science 10 (1973) 745-766.

16 J.W. Hntchinson, M. Mear and J.R. Rice, ASME Journal of Applied Mechanics 54 (1988) 828-832.

17 B.M. Malysher and R.L. Salganik, International Journal of Fracture Mechanics, 1 (1965) 114-128.

18 F. Exdogan and G.D. Gupta, International Journal of Solids \& Structures 7 (1971) $1089-1107$.

19 J. Dundurs, in Mathematical Theory of Dislocations, American Society of Mechanical Engineering, New York (1969) 70-115. 


\section{Appendix}

For dislocation $b_{z}$ lying at interface $(0,0)$

$$
\begin{aligned}
& f_{1}(\xi)=\frac{C b_{z}}{4 \mu_{1}}\left[\beta+(1+\beta) h_{1} \xi\right] e^{-h_{1} \xi} \\
& f_{2}(\xi)=-\frac{C b_{z}}{4 \mu_{1}}\left[1-(1+\beta) h_{1} \xi\right] e^{-h_{1} \xi} \\
& f_{3}(\xi)=-\frac{C b_{z}}{4 \mu_{2}}\left[\beta-(1-\beta) h_{2} \xi\right] e^{-h_{2} \xi} \\
& f_{4}(\xi)=-\frac{C b_{z}}{4 \mu_{2}}\left[1-(1-\beta) h_{2} \xi\right] e^{-h_{2} \xi} .
\end{aligned}
$$

For dislocation $b_{y}$ lying at interface $(0,0)$

$$
\begin{aligned}
& f_{1}(\xi)=\frac{C b_{y}}{4 \mu_{1}}\left[1+(1+\beta) h_{1} \xi\right] e^{-h_{1} \xi} \\
& f_{2}(\xi)=-\frac{C b_{y}}{4 \mu_{1}}\left[\beta-(1+\beta) h_{1} \xi\right] e^{-h_{1} \xi} \\
& f_{f}(\xi)=\frac{C b_{y}}{4 \mu_{2}}\left[1+(1-\beta) h_{2} \xi\right] e^{-h_{2} \xi} \\
& f_{4}(\xi)=-\frac{C b_{y}}{4 \mu_{2}}\left[\beta+(1-\beta) h_{2} \xi\right] e^{-h_{2} \xi} .
\end{aligned}
$$

For dislocation $b_{4}$ lying at $(0, \zeta)$ with $\zeta<0$ 


$$
\begin{aligned}
& f_{1}(\xi)=\frac{C b_{2}}{4 \mu_{1}}\left[\beta+\left(h_{1}-\zeta\right) \xi+\left(h_{1}+\zeta\right) \beta \xi\right] e^{-\left(h_{2}-\zeta\right) \xi} \\
& f_{2}(\xi)=-\frac{C b_{z}}{4 \mu_{1}}\left[1-(1+\beta)\left(h_{1}-\zeta\right) \xi-2 \beta \zeta \xi\right] e^{-\left(h_{1}-\zeta \xi\right.} \\
& f_{3}(\xi)=-\frac{C b_{2}}{4 \mu_{2}(1+\alpha)}\left[\left(1-\beta^{2}\right)\left(h_{2}+\zeta\right) \xi e^{-\left(h_{2}+\zeta\right) \xi}\right. \\
&+\frac{C b_{3}}{4 \mu_{2}(1+\alpha)}\left[\beta(1+\alpha)-(\alpha-\beta)(1-\beta)\left(h_{3}+\zeta\right) \xi\right. \\
&\left.-2(\alpha-\beta)(1-\beta) h_{2} \zeta \xi^{2}\right] e^{-\left(h_{2}-\zeta\right) \xi} \\
& f_{4}(\xi)=-\frac{C b_{z}\left(1-\beta^{2}\right)}{4 \mu_{2}(1+\alpha)}\left[1-\left(h_{2}+\zeta\right) \xi\right] e^{-\left(h_{2}+\zeta\right) \xi} \\
&-\frac{C b_{2}}{4 \mu_{2}(1+\alpha)}\left[\left(\alpha+\beta^{2}\right)-(\alpha-\beta)(1-\beta)\left(h_{2}-\zeta\right) \xi\right. \\
&\left.-2(\alpha-\beta)(1-\beta) \zeta h_{2} \xi^{2}\right] e^{-\left(h_{2}-\zeta \xi\right.} .
\end{aligned}
$$

For four-paint bending

$$
\begin{array}{ll}
f_{1}(\xi)=-\frac{p}{4 \mu_{1}} \cos \xi d, & f_{2}(\xi)=0 \\
f_{3}(\xi)=-\frac{p}{4 \mu_{2}} \cos \xi e, & f_{4}(\xi)=0 .
\end{array}
$$


Table 1 Stress intensity factors for edge crack under tension along nentral axas $\left(c / h_{2}=\right.$ 0.5).

\begin{tabular}{|c|c|c|c|c|c|c|c|c|c|c|}
\hline \multicolumn{11}{|c|}{$K / \sigma^{(2)} \sqrt{\pi c / 2}$} \\
\hline \multirow[b]{2}{*}{$h_{1} / h_{2}$} & \multirow[b]{2}{*}{$\beta$} & \multicolumn{9}{|c|}{$\alpha$} \\
\hline & & -0.8 & -0.6 & -0.4 & -0.2 & 0.0 & 0.2 & 0.4 & 0.6 & 0.8 \\
\hline \multirow{9}{*}{0.1} & -0.4 & 3.891 & 3.786 & & & & & & & \\
\hline & -0.3 & 3.892 & 3.788 & 3.081 & 3.571 & & & & & \\
\hline & -0.2 & 3.893 & 3.791 & 3.685 & 3.576 & 3.468 & $3.34:$ & & & \\
\hline & -0.1 & 3.894 & 3.793 & 3.688 & 3.581 & 3.468 & 3.355 & 3.231 & 3.102 & \\
\hline & 0.0 & 3.895 & 3.795 & 3.692 & 3.585 & 3.474 & 3.360 & 3.240 & 3.112 & 2.967 \\
\hline & 0.1 & & & 3.696 & 3.589 & 3.480 & 3.366 & 3.248 & 3.122 & 2.979 \\
\hline & 0.2 & & & & & 3.48 & 3.375 & 3.255 & 3.131 & 2.991 \\
\hline & 0.3 & & & & & & & 3.262 & 3.139 & 3.001 \\
\hline & 0.4 & & & & & & & & & 3.011 \\
\hline
\end{tabular}

\begin{tabular}{|c|c|c|c|c|c|c|c|c|c|c|}
\hline \multicolumn{11}{|c|}{$K / \sigma^{(2)} \sqrt{\pi c / 2}$} \\
\hline \multirow[b]{2}{*}{$h_{1} / h_{2}$} & \multirow[b]{2}{*}{$\beta$} & \multicolumn{9}{|c|}{$\alpha$} \\
\hline & & -0.8 & -0.6 & -0.4 & -0.2 & 0.0 & 0.2 & 0.4 & 0.6 & 0.8 \\
\hline \multirow{9}{*}{1} & -0.4 & 2.782 & 2.435 & & & & & & & \\
\hline & -0.3 & 2.828 & 2.481 & 2.284 & 2.146 & & & & & \\
\hline & -0.2 & 2.866 & 2.520 & 2.322 & 2.181 & 2.066 & 1.964 & & & \\
\hline & -0.1 & 2.900 & 2.554 & 2.354 & 2.211 & 2.094 & 1.989 & 1.886 & 1.773 & \\
\hline & 0.0 & 2.929 & 2.584 & 2.383 & 2.237 & 2.117 & 2.010 & 1.903 & 1.786 & 1.635 \\
\hline & 0.1 & & & 2.408 & 2.260 & 2.138 & 2.027 & 1.917 & 1.794 & 1.697 \\
\hline & 0.2 & & & & & 2.155 & 2.041 & 1.927 & 1.800 & 1.636 \\
\hline & 0.3 & & & & & & & 1.933 & 1.801 & 1.629 \\
\hline & 0.4 & & & & & & & & & 1.618 \\
\hline
\end{tabular}

\begin{tabular}{|c|c|c|c|c|c|c|c|c|c|c|}
\hline \multicolumn{11}{|c|}{$K / \sigma^{(2)} \sqrt{\pi c / 2}$} \\
\hline \multirow[b]{2}{*}{$h_{1} / h_{2}$} & \multirow[b]{2}{*}{$\boldsymbol{\beta}$} & \multicolumn{9}{|c|}{$\alpha$} \\
\hline & & -0.8 & -0.6 & -0.4 & -0.2 & 0.0 & 0.2 & 0.4 & 0.6 & 0.8 \\
\hline \multirow{9}{*}{10} & -0.4 & 2.117 & 1.894 & & & & & & & \\
\hline & -0.3 & 2.137 & 1.910 & 1.774 & 1.678 & & & & & \\
\hline & -0.2 & 2.149 & 1.920 & 1.783 & 1.685 & 1.610 & 1.549 & & & \\
\hline & -0.1 & 2.157 & 1.924 & 1.786 & 1.887 & 1.611 & 1.549 & 1.498 & 1.453 & \\
\hline & 0.0 & 2.158 & 1.924 & 1.784 & 1.684 & 1.608 & 1.545 & 1.493 & 1.449 & 1.410 \\
\hline & 0.1 & & & 1.778 & 1.677 & 1.600 & 1.537 & 1.485 & 1.440 & 1.401 \\
\hline & 0.2 & & & & & 1.588 & 1.525 & 1.472 & 1.427 & 1.388 \\
\hline & 0.3 & & & & & & & 1.454 & 1.409 & 1.370 \\
\hline & 0.4 & & & & & & & & & 1.346 \\
\hline
\end{tabular}


Table 2 Stress intensity factors for edge crack under pure bending $\left(c / h_{2}=0.5\right)$.

\begin{tabular}{||c|c|c|c|c|c|c|c|c|c|c||}
\hline \multicolumn{10}{|c||}{$K / \sigma_{\max } \sqrt{\pi c / 2}$} \\
\hline \multirow{3}{*}{$h_{1} / h_{2}$} & $\beta$ & -0.8 & -0.6 & -0.4 & -0.2 & 0.0 & 0.2 & 0.4 & 0.6 & 0.8 \\
\hline & -0.4 & 2.074 & 2.032 & & & & & & & \\
& -0.3 & 2.074 & 2.034 & 1.996 & 1.960 & & & & & \\
& -0.2 & 2.075 & 2.056 & 1.988 & 1.964 & 1.934 & 1.913 & & & \\
& -0.1 & 2.076 & 2.037 & 2.000 & 1.967 & 1.998 & 1.918 & 1.910 & 1.924 & \\
& 0.1 & 2.077 & 2.038 & 2.002 & 1.969 & 1.942 & 1.922 & 1.916 & 1.931 & 1.982 \\
& 0.1 & & & 2.004 & 1.972 & 1.945 & 1.927 & 1.921 & 1.938 & 1.991 \\
& 0.2 & & & & & 1.949 & 1.931 & 1.926 & 1.944 & 1.999 \\
& 0.3 & & & & & & & 1.931 & 1.950 & 2.008 \\
& 0.4 & & & & & & & & & 2.015 \\
\hline
\end{tabular}

\begin{tabular}{||c|c|c|c|c|c|c|c|c|c|c||}
\hline \multicolumn{10}{|c|}{$K / \sigma_{\max } \sqrt{\pi c / 2}$} \\
\hline \multirow{3}{*}{$h_{1} / h_{2}$} & $\beta$ & -0.8 & -0.6 & -0.4 & -0.2 & 0.0 & 0.2 & 0.4 & 0.6 & 0.8 \\
\hline & -0.4 & 1.594 & 1.509 & & & & & & & \\
& -0.3 & 1.625 & 1.543 & 1.510 & 1.487 & & & & & \\
& -0.2 & 1.652 & 1.572 & 1.539 & 1.514 & 1.488 & 1.457 & & & \\
& -0.1 & 1.675 & 1.597 & 1.564 & 1.538 & 1.511 & 1.477 & 1.434 & 1.373 & \\
& 0.0 & 1.695 & 1.619 & 1.585 & 1.559 & 1.530 & 1.495 & 1.448 & 1.383 & 1.283 \\
& 0.1 & & & 1.605 & 1.577 & 1.547 & 1.509 & 1.460 & 1.391 & 1.285 \\
& 0.2 & & & & & 1.561 & 1.521 & 1.468 & 1.396 & 1.284 \\
& 0.3 & & & & & & & 1.474 & 1.397 & 1.278 \\
& 0.4 & & & & & & & & & 1.269 \\
\hline
\end{tabular}

\begin{tabular}{||c|c|c|c|c|c|c|c|c|c|c||}
\hline \multicolumn{10}{|c||}{$K / \sigma_{\max } \sqrt{\pi c / 2}$} \\
\hline \hline \multirow{6}{*}{$h_{1} / h_{2}$} & $\beta$ & -0.8 & -0.6 & -0.4 & -0.2 & 0.0 & 0.2 & 0.4 & 0.6 & 0.8 \\
\hline & -0.4 & 1.945 & 1.771 & & & & & & & \\
& -0.3 & 1.963 & 1.786 & 1.669 & 1.583 & & & & & \\
& -0.2 & 1.975 & 1.796 & 1.677 & 1.590 & 1.521 & 1.464 & & & \\
& -0.1 & 1.982 & 1.800 & 1.680 & 1.592 & 1.522 & 1.465 & 1.417 & 1.375 & \\
10 & 0.0 & 1.984 & 1.800 & 1.679 & 1.589 & 1.519 & 1.461 & 1.413 & 1.371 & 1.334 \\
& 0.1 & & & 1.673 & 1.582 & 1.512 & 1.464 & 1.405 & 1.363 & 1.326 \\
& 0.2 & & & & & 1.500 & 1.441 & 1.392 & 1.350 & 1.313 \\
& 0.3 & & & & & & & 1.375 & 1.333 & 1.296 \\
& 0.4 & & & & & & & & & 1.273 \\
\hline
\end{tabular}


Table 3 Singularity for edge crack whose tip touches the interface.

\begin{tabular}{|c|c|c|c|c|c|c|c|c|c|}
\hline \multicolumn{10}{|c|}{$\lambda$} \\
\hline \multirow[b]{2}{*}{$\boldsymbol{\beta}$} & \multicolumn{9}{|c|}{$\alpha$} \\
\hline & -0.8 & -0.6 & -0.4 & -0.2 & 0.0 & 0.2 & 0.4 & 0.6 & 0.8 \\
\hline-0.4 & 0.7321 & 0.6449 & & & & & & & \\
\hline-0.3 & 0.7994 & 0.6507 & 0.5949 & 0.5567 & & & & & \\
\hline-0.2 & 0.7438 & 0.6533 & 0.5946 & 0.5533 & 0.5230 & 0.5000 & & & \\
\hline-0.1 & 0.7456 & 0.6529 & 0.5912 & 0.5467 & 0.5135 & 0.4881 & 0.4682 & 0.4523 & \\
\hline 0.0 & 0.7450 & 0.6495 & 0.5843 & 0.5364 & 0.5000 & 0.4718 & 0.4496 & 0.4318 & 0.4173 \\
\hline 0.1 & & & 0.5734 & 0.5213 & 0.4812 & 0.4498 & 0.4249 & 0.4049 & 0.3887 \\
\hline 0.2 & & & & & 0.4551 & 0.4196 & 0.3913 & 0.3687 & 0.3504 \\
\hline 0.3 & & & & & & & 0.3435 & 0.3173 & 0.2963 \\
\hline 0.4 & & & & & & & & & 0.2114 \\
\hline
\end{tabular}


Table 4 Stress intensity factors for edge crack whose tip tonches the interface (tension along neutral axig).

\begin{tabular}{|c|c|c|c|c|c|c|c|c|c|c|}
\hline \multicolumn{11}{|c|}{$K / \sigma^{(2)} \sqrt{\pi}\left(h_{2} / 2\right)^{\lambda}$} \\
\hline \multirow[b]{2}{*}{$h_{1} / h_{2}$} & \multirow[b]{2}{*}{$\boldsymbol{\beta}$} & \multicolumn{9}{|c|}{$\alpha$} \\
\hline & & -0.8 & -0.6 & -0.4 & -0.2 & 0.0 & 0.2 & 0.4 & 0.6 & 0.8 \\
\hline \multirow{9}{*}{0.1} & -0.4 & 42.29 & 64.73 & & & & & & & \\
\hline & -0.3 & 36.34 & 57.24 & 65.37 & 66.57 & & & & & \\
\hline & -0.2 & 31.86 & 51.88 & 59.99 & 61.77 & 59.26 & 53.75 & & & \\
\hline & -0.1 & 28.41 & 48.00 & 56.27 & 58.63 & 56.83 & 52.00 & 44.82 & 36.58 & \\
\hline & 0.0 & 25.70 & 45.26 & 53.86 & 56.87 & 55.76 & 51.48 & 44.67 & 35.59 & 23.71 \\
\hline & 0.1 & & & 52.70 & 56.49 & 56.14 & 52.38 & 45.77 & 36.57 & 24.27 \\
\hline & 0.2 & & & & & 58.45 & 55.26 & 48.70 & 39.02 & 25.72 \\
\hline & 0.3 & & & & & & & 55.06 & 44.28 & 28.90 \\
\hline & 0.4 & & & & & & & & & 36.76 \\
\hline
\end{tabular}

\begin{tabular}{||c|c|c|c|c|c|c|c|c|c|c||}
\hline \multicolumn{10}{|c||}{$K / \sigma^{(2)} \sqrt{\pi}\left(h_{2} / 2\right)^{\lambda}$} \\
\hline \multirow{3}{*}{$h_{1} / h_{2}$} & $\beta$ & -0.8 & -0.6 & -0.4 & -0.2 & 0.0 & 0.2 & 0.4 & 0.6 & 0.8 \\
\hline & -0.4 & 6.209 & 6.483 & & & & & & & \\
& -0.3 & 5.631 & 5.901 & 5.625 & 5.120 & & & & & \\
& -0.2 & 5.182 & 5.442 & 5.238 & 4.819 & 4.300 & 3.741 & & & \\
& -0.1 & 4.820 & 5.074 & 4.927 & 4.579 & 4.123 & 3.613 & 3.078 & 2.524 & \\
1 & 0.0 & 4.523 & 4.775 & 4.679 & 4.393 & 3.993 & 3.526 & 3.020 & 2.487 & 1.918 \\
& 0.1 & & & 4.488 & 4.262 & 3.914 & 3.485 & 3.004 & 2.484 & 1.921 \\
& 0.2 & & & & & 3.904 & 3.512 & 3.049 & 2.532 & 1.963 \\
& 0.3 & & & & & & & 3.210 & 2.681 & 2.082 \\
& 0.4 & & & & & & & & & 2.417 \\
\hline
\end{tabular}

\begin{tabular}{|c|c|c|c|c|c|c|c|c|c|c||}
\hline \multicolumn{10}{|c|}{$K / \sigma^{(2)} \sqrt{\pi}\left(h_{2} / 2\right)^{\lambda}$} \\
\hline \multirow{3}{*}{$h_{1} / h_{2}$} & $\beta$ & -0.8 & -0.6 & -0.4 & -0.2 & 0.0 & 0.2 & 0.4 & 0.6 & 0.8 \\
\hline & -0.4 & 2.465 & 2.351 & & & & & & & \\
& -0.3 & 2.286 & 2.189 & 2.064 & 1.931 & & & & & \\
& -0.2 & 2.149 & 2.063 & 1.962 & 1.851 & 1.738 & 1.629 & & & \\
& -0.1 & 2.042 & 1.865 & 1.883 & 1.791 & 1.693 & 1.595 & 1.500 & 1.413 & \\
& 0.0 & 1.958 & 1.890 & 1.825 & 1.750 & 1.666 & 1.577 & 1.489 & 1.405 & 1.326 \\
& 0.1 & & & 1.790 & 1.732 & 1.662 & 1.582 & 1.499 & 1.416 & 1.596 \\
& 0.2 & & & & & 1.689 & 1.621 & 1.542 & 1.458 & 1.375 \\
& 0.3 & & & & & & & 1.649 & 1.563 & 1.471 \\
& 0.4 & & & & & & & & & 1.726 \\
\hline
\end{tabular}


Table 5 Stress intensity factors for edge crack whose tip touches the interface (pure bending).

\begin{tabular}{|c|c|c|c|c|c|c|c|c|c|c|}
\hline \multicolumn{11}{|c|}{$K^{\prime} \sigma_{\max } \sqrt{\pi}\left(h_{2} / 2\right)^{\lambda}$} \\
\hline \multirow[b]{2}{*}{$h_{1} / h_{2}$} & \multirow[b]{2}{*}{$\boldsymbol{\beta}$} & \multicolumn{9}{|c|}{$\alpha$} \\
\hline & & -0.8 & -0.6 & -0.4 & -0.2 & 0.0 & 0.2 & 0.4 & 0.6 & 0.8 \\
\hline \multirow{9}{*}{0.1} & -0.4 & 12.59 & 20.29 & & & & & & & \\
\hline & -0.3 & 10.7 & 17.87 & 21.27 & 22.67 & & & & & \\
\hline & -0.2 & 9.280 & 16.13 & 19.47 & 20.99 & 21.25 & 20.56 & & & \\
\hline & -0.1 & 8.165 & 14.86 & 18.20 & 19.87 & 20.39 & 19.85 & 18.56 & 16.35 & \\
\hline & 0.0 & 7.279 & 13.94 & 17.36 & 19.22 & 19.90 & 19.61 & 18.46 & 16.33 & 12.51 \\
\hline & 0.1 & & & 16.93 & 19.03 & 19.98 & 19.90 & 18.87 & 16.74 & 12.77 \\
\hline & 0.2 & & & & & 20.73 & 20.94 & 20.02 & 17.81 & 13.50 \\
\hline & 0.3 & & & & & & & 22.56 & 20.14 & 15.10 \\
\hline & 0.4 & & & & & & & & & 19.11 \\
\hline
\end{tabular}

\begin{tabular}{||c|c|c|c|c|c|c|c|c|c|c||}
\hline \multicolumn{10}{|c|}{$K / \sigma_{\max } \sqrt{\pi}\left(h_{2} / 2\right)^{\lambda}$} \\
\hline \hline \multirow{6}{*}{$h_{1} / h_{2}$} & $\beta$ & -0.8 & -0.6 & -0.4 & -0.2 & 0.0 & 0.2 & 0.4 & 0.6 & 0.8 \\
\hline & -0.4 & 1.739 & 2.478 & & & & & & & \\
& -0.3 & 1.538 & 2.215 & 2.536 & 2.605 & & & & & \\
& -0.2 & 1.379 & 2.004 & 2.324 & 2.417 & 2.353 & 2.182 & & & \\
& -0.1 & 1.247 & 1.890 & 2.147 & 2.260 & 2.223 & 2.077 & 1.855 & 1.572 & \\
& 0.0 & 1.134 & 1.683 & 1.999 & 2.130 & 2.116 & 1.994 & 1.790 & 1.522 & 1.188 \\
& 0.1 & & & 1.874 & 2.024 & 2.039 & 1.992 & 1.745 & 1.487 & 1.160 \\
& 0.2 & & & & & 1.979 & 1.900 & 1.727 & 1.475 & 1.148 \\
& 0.3 & & & & & & & 1.758 & 1.505 & 1.168 \\
& 0.4 & & & & & & & & & 1.266 \\
\hline
\end{tabular}

\begin{tabular}{|c|c|c|c|c|c|c|c|c|c|c||}
\hline \multicolumn{10}{|c|}{$K / \sigma_{\max } \sqrt{\pi}\left(h_{2} / 2\right)^{\lambda}$} \\
\hline \multirow{3}{*}{$h_{1} / h_{2}$} & $\beta$ & -0.8 & -0.6 & -0.4 & -0.2 & 0.0 & 0.2 & 0.4 & 0.6 & 0.8 \\
\hline & -0.4 & 2.095 & 2.077 & & & & & & & \\
& -0.3 & 1.937 & 1.927 & 1.841 & 1.734 & & & & & \\
& -0.2 & 1.815 & 1.810 & 1.741 & 1.656 & 1.561 & 1.468 & & & \\
& -0.1 & 1.719 & 1.718 & 1.663 & 1.596 & 1.515 & 1.431 & 1.350 & 1.274 & \\
& 0.0 & 1.643 & 1.647 & 1.611 & 1.554 & 1.485 & 1.409 & 1.394 & 1.260 & 1.191 \\
& 0.1 & & & 1.573 & 1.531 & 1.474 & 1.406 & 1.355 & 1.263 & 1.194 \\
& 0.2 & & & & & 1.489 & 1.432 & 1.364 & 1.292 & 1.220 \\
& 0.3 & & & & & & & 1.447 & 1.372 & 1.293 \\
& 0.4 & & & & & & & & & 1.497 \\
\hline
\end{tabular}




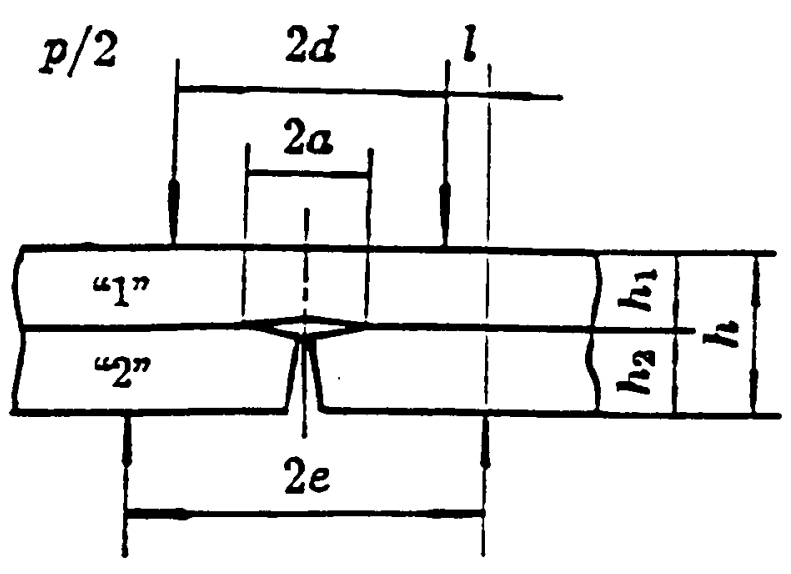

(a)

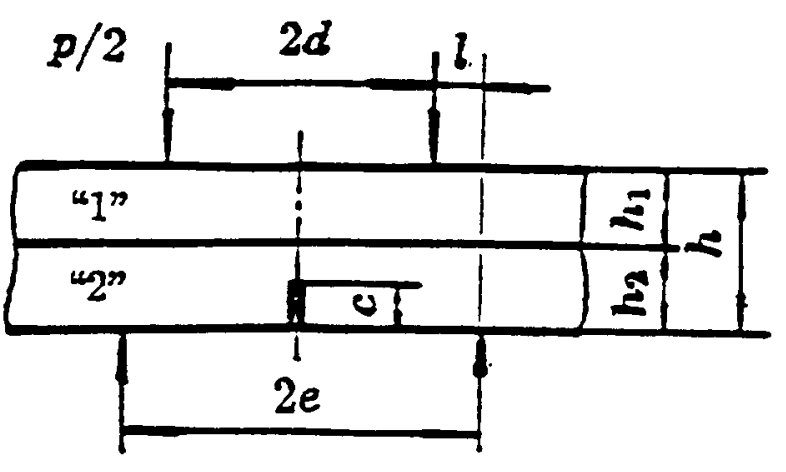

(c)

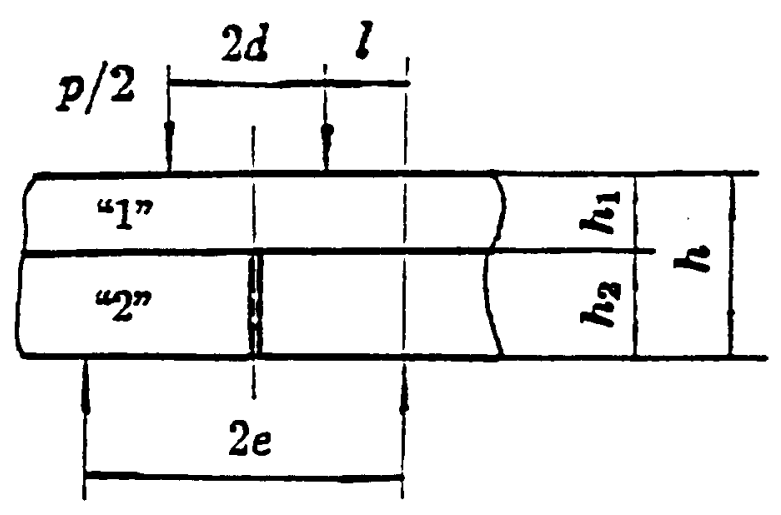

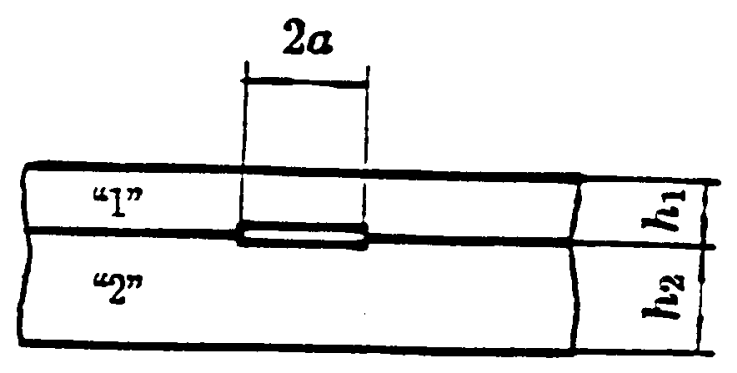

(b)

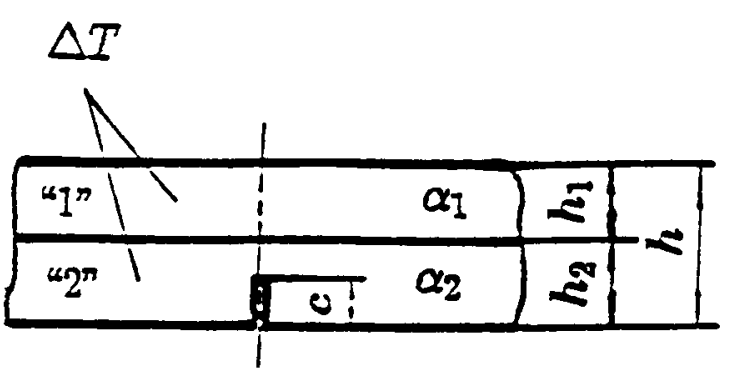

(d)

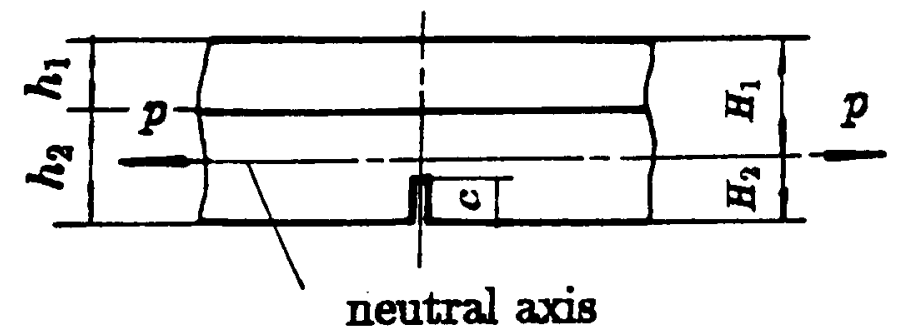

Fig. 1 Crack configurations for the bimaterial strip.

(e) 


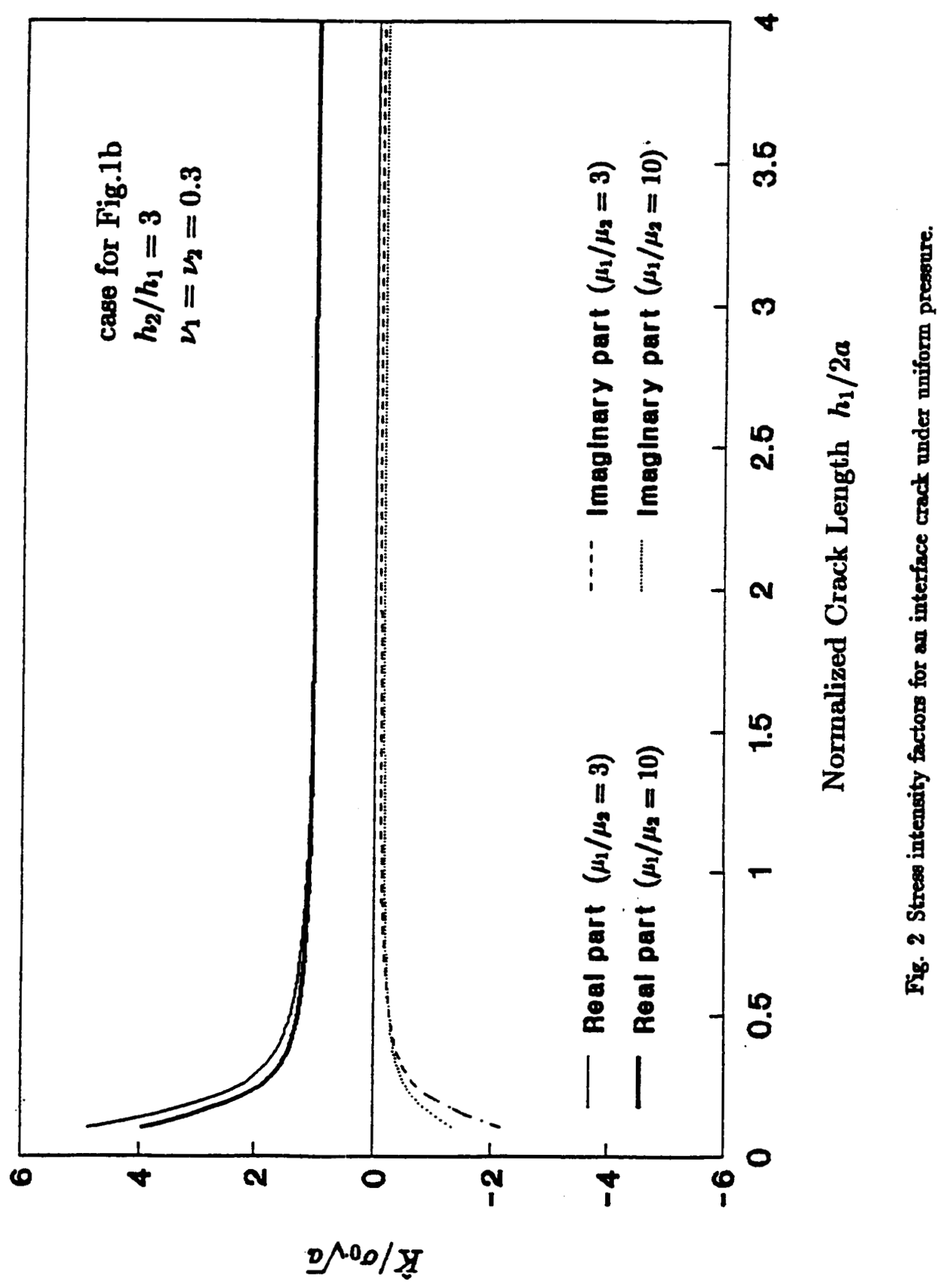




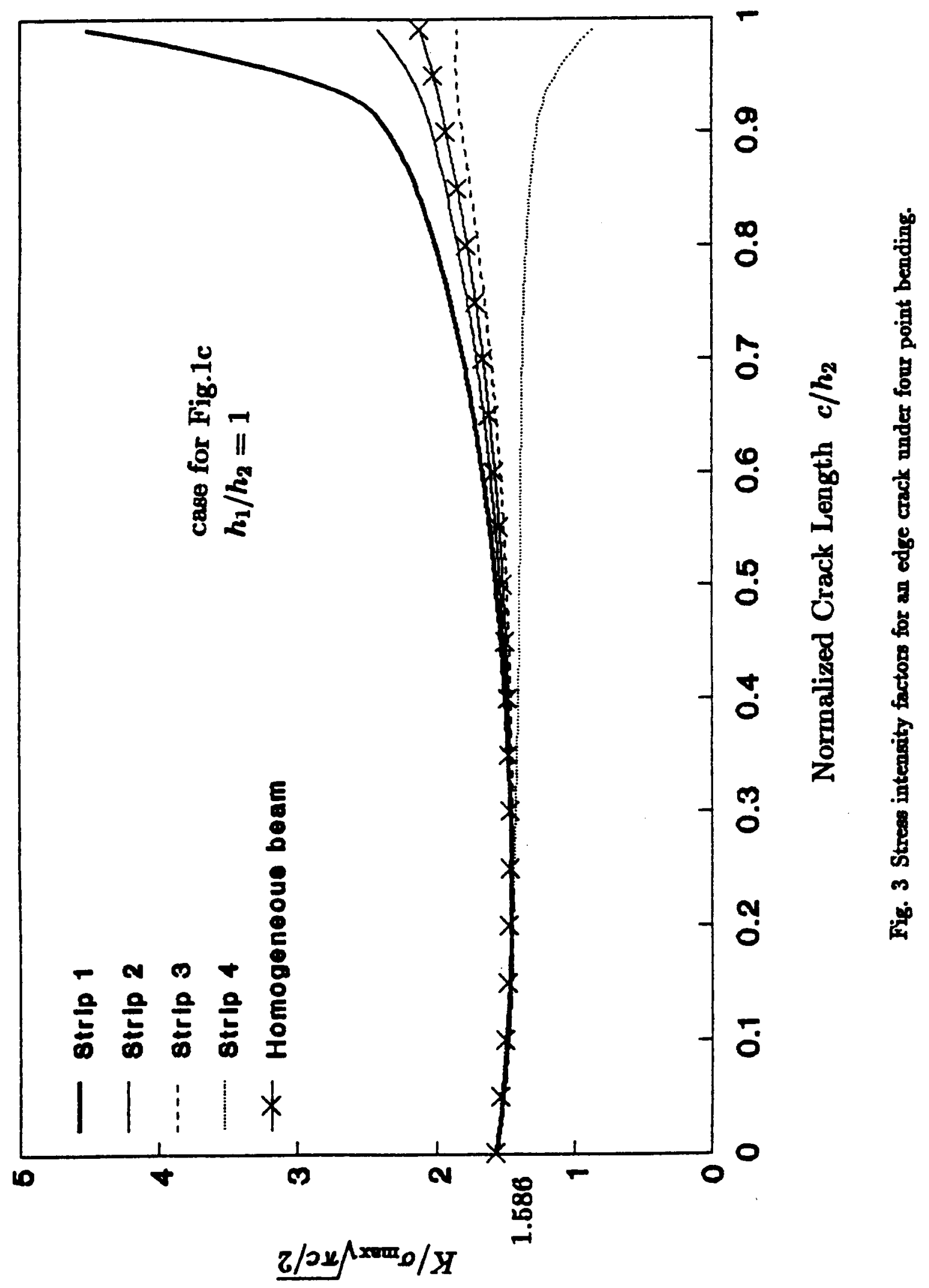




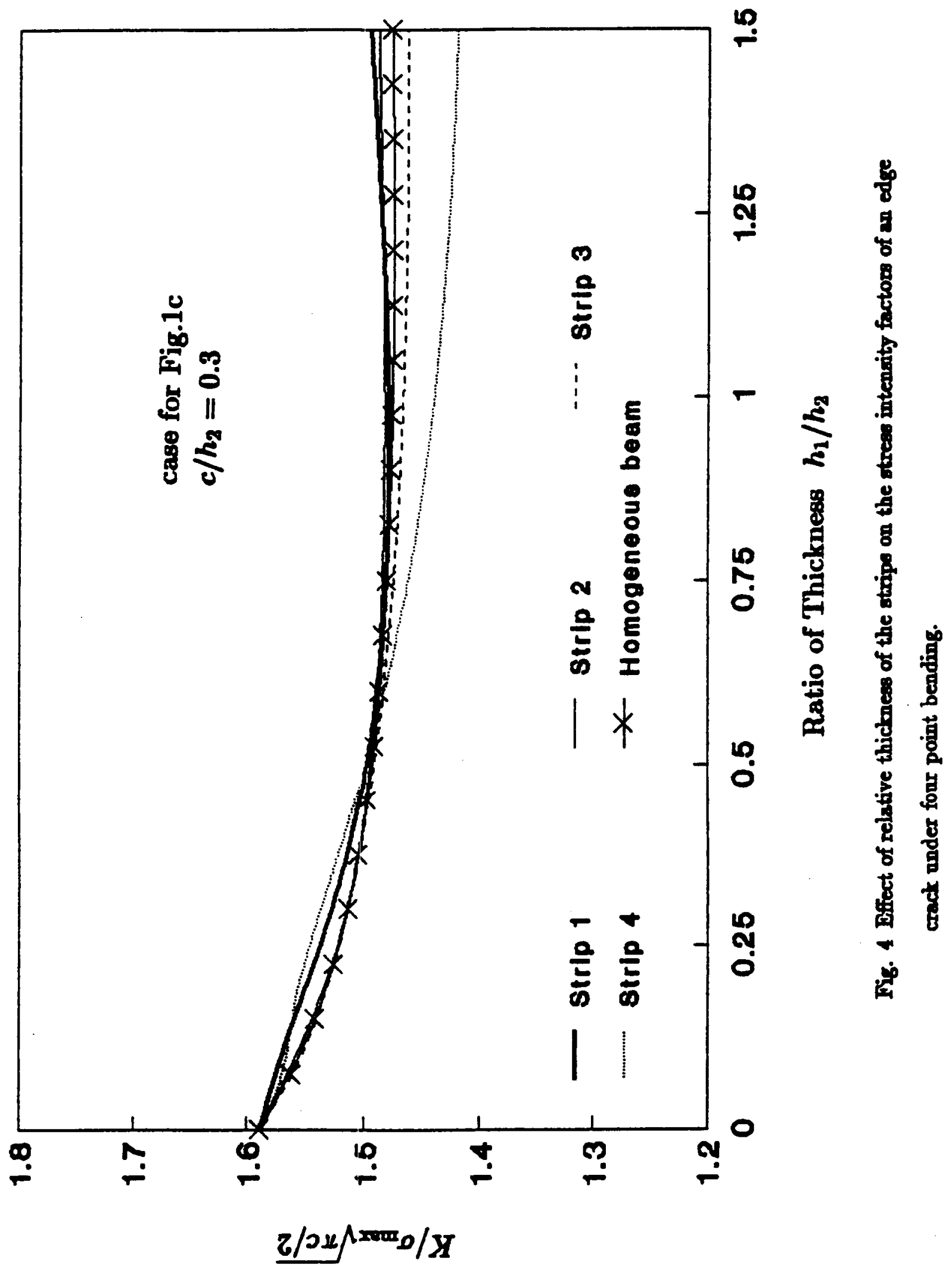




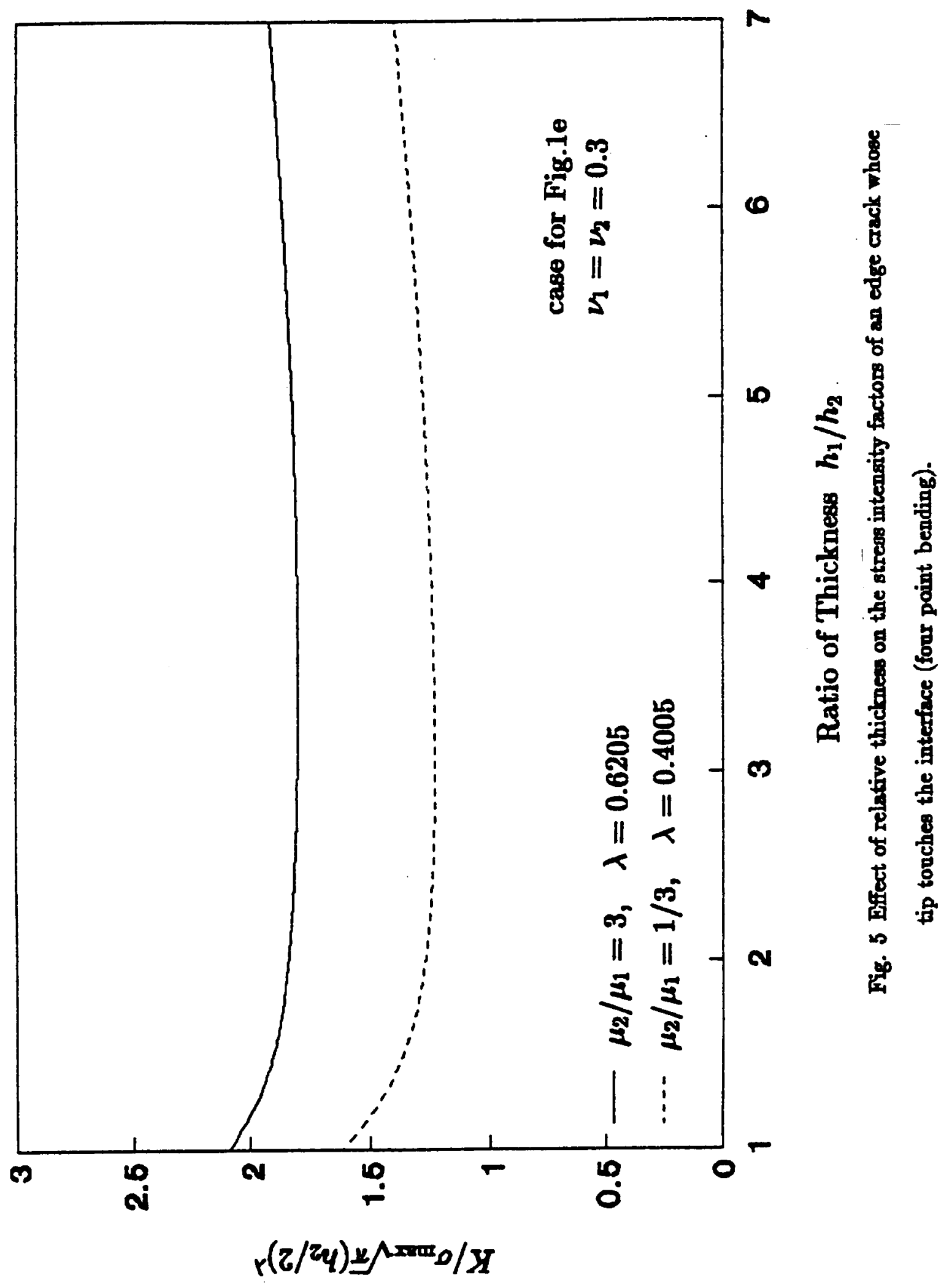




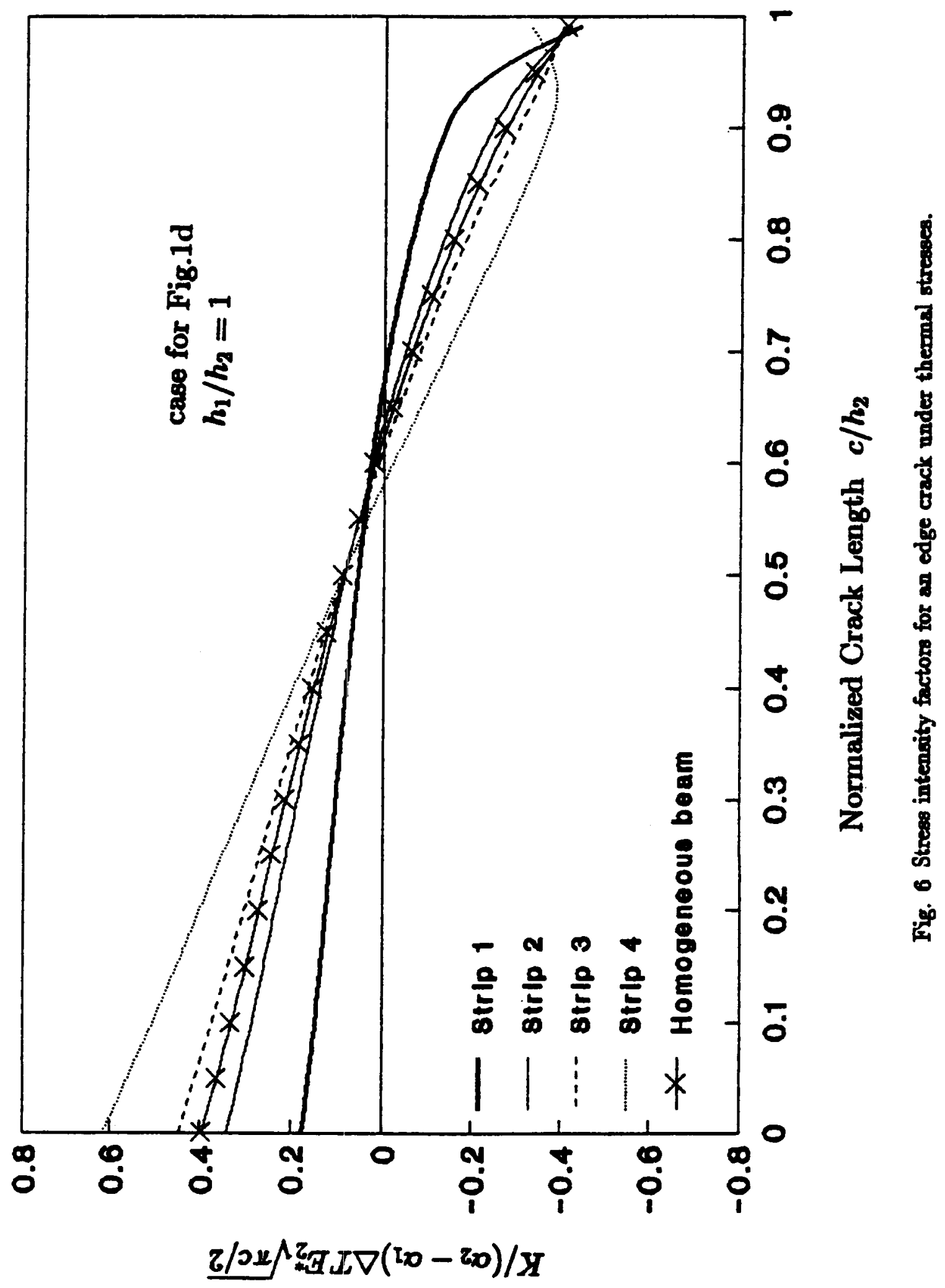




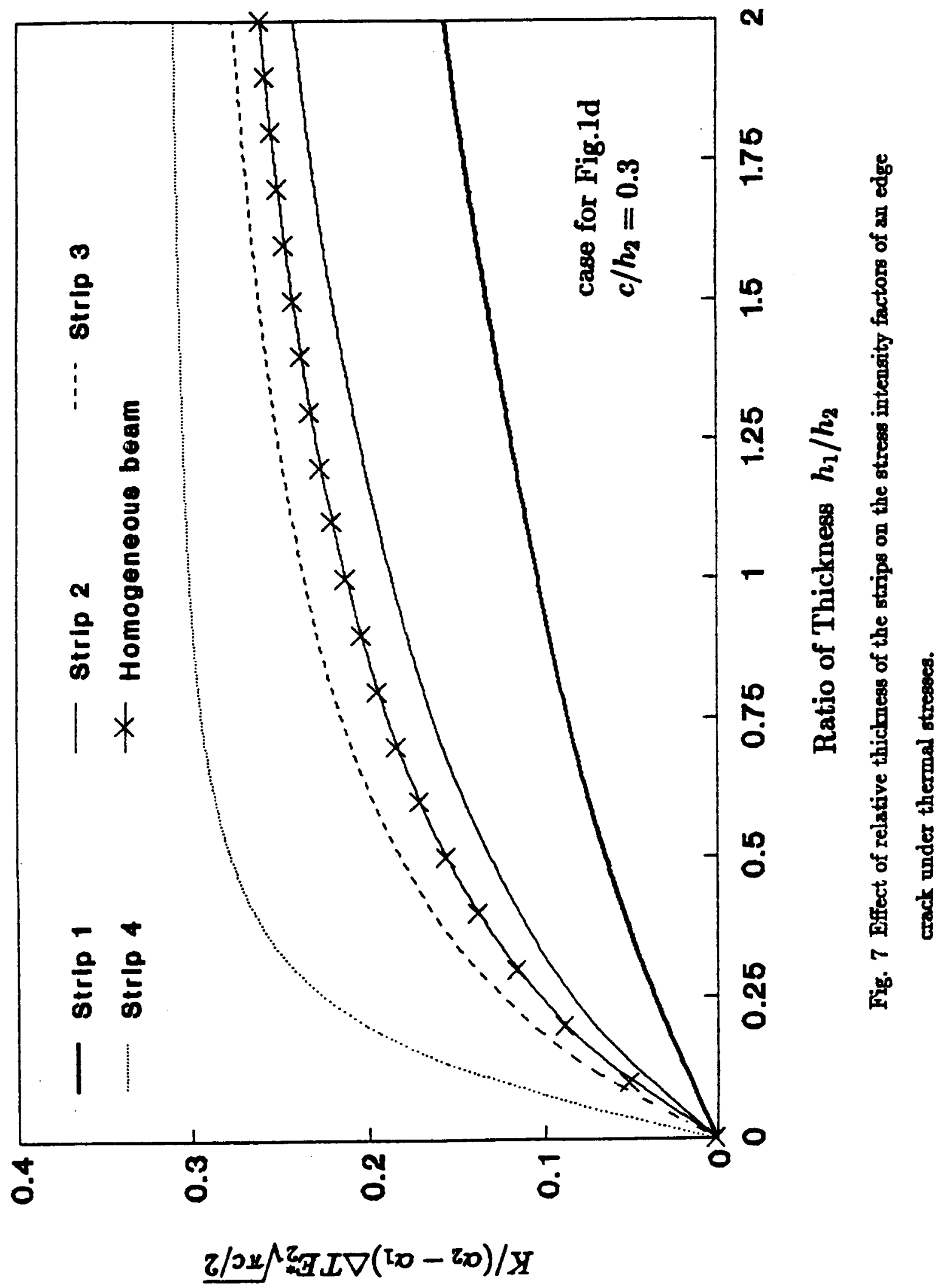




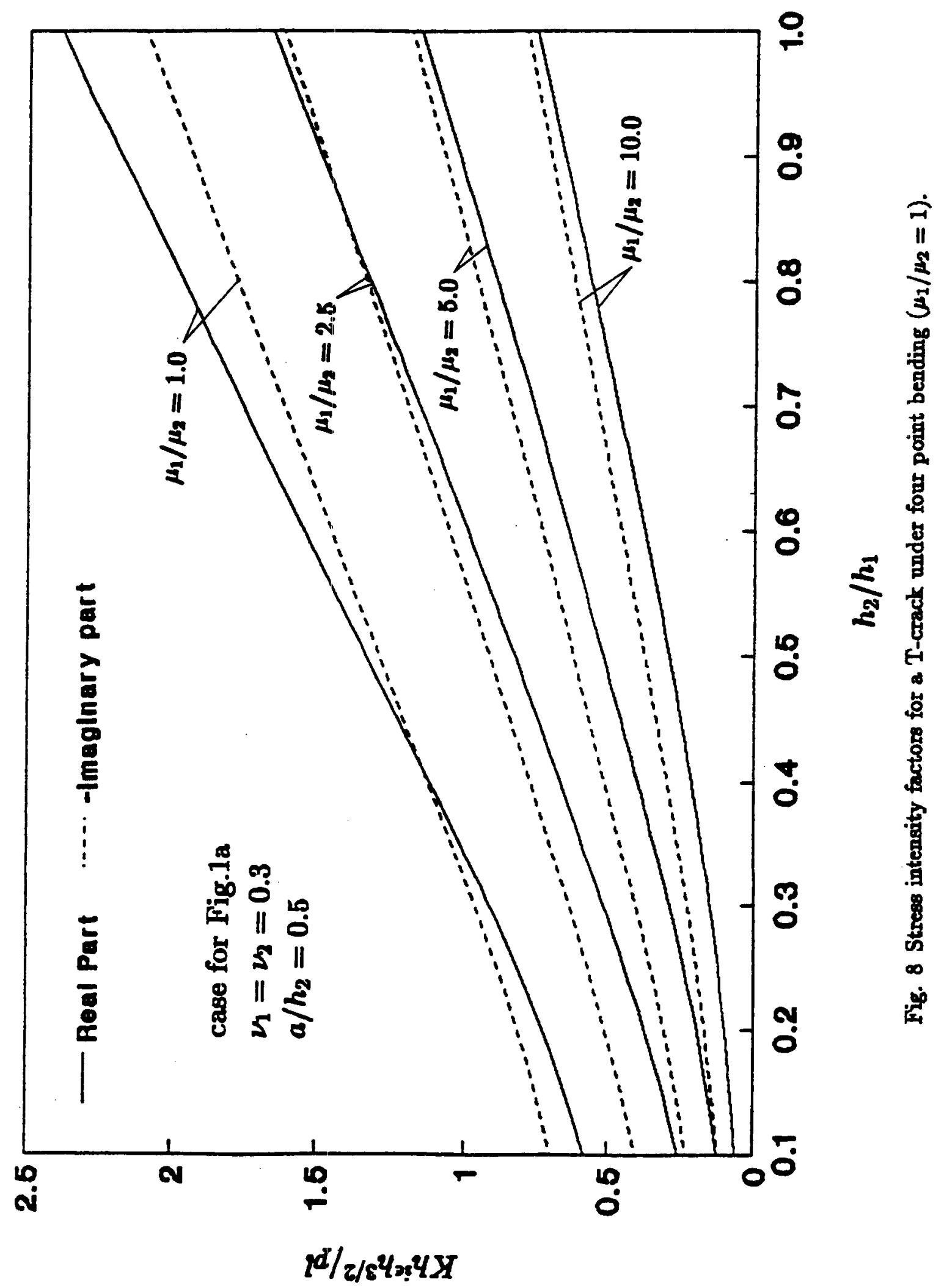




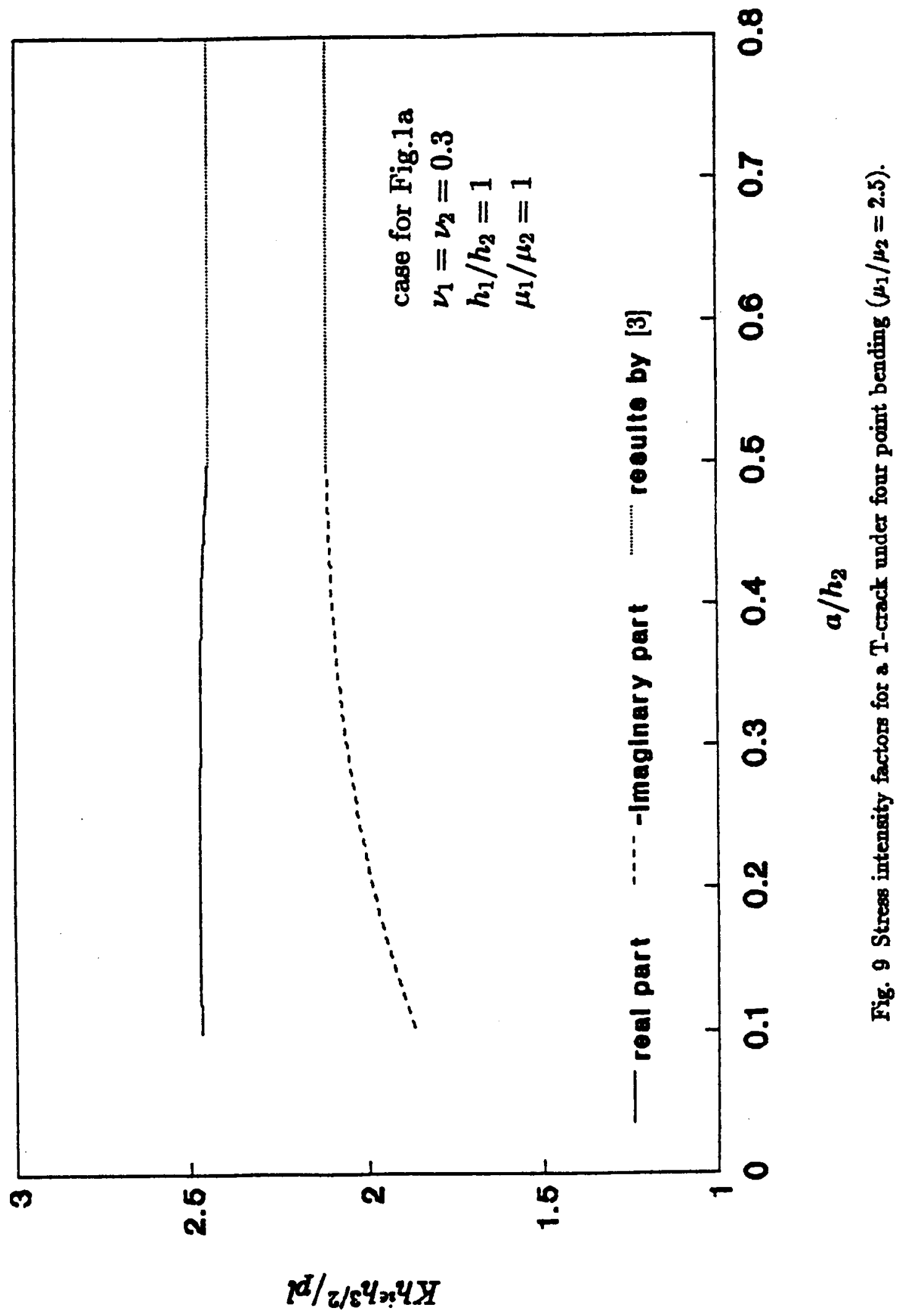




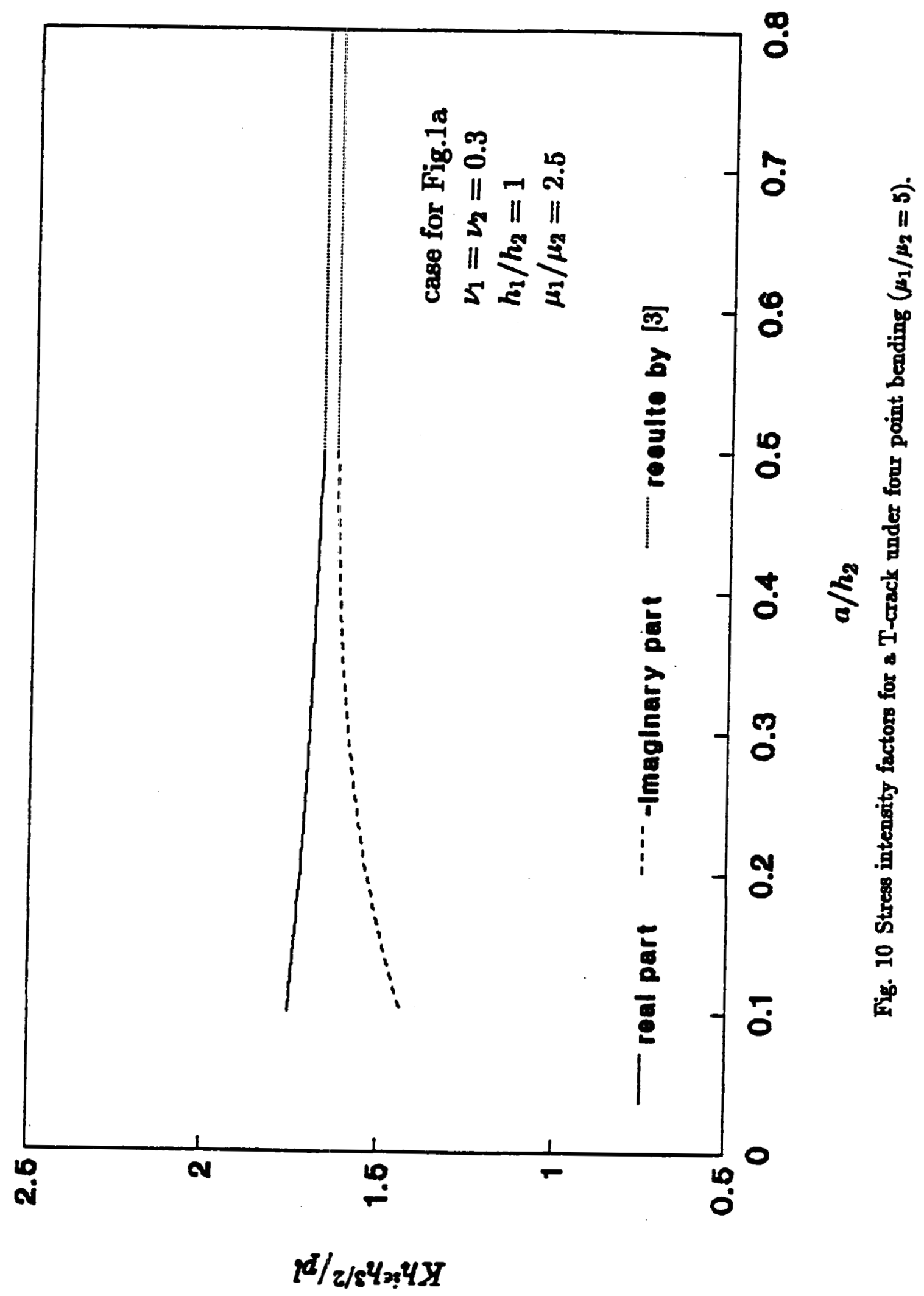




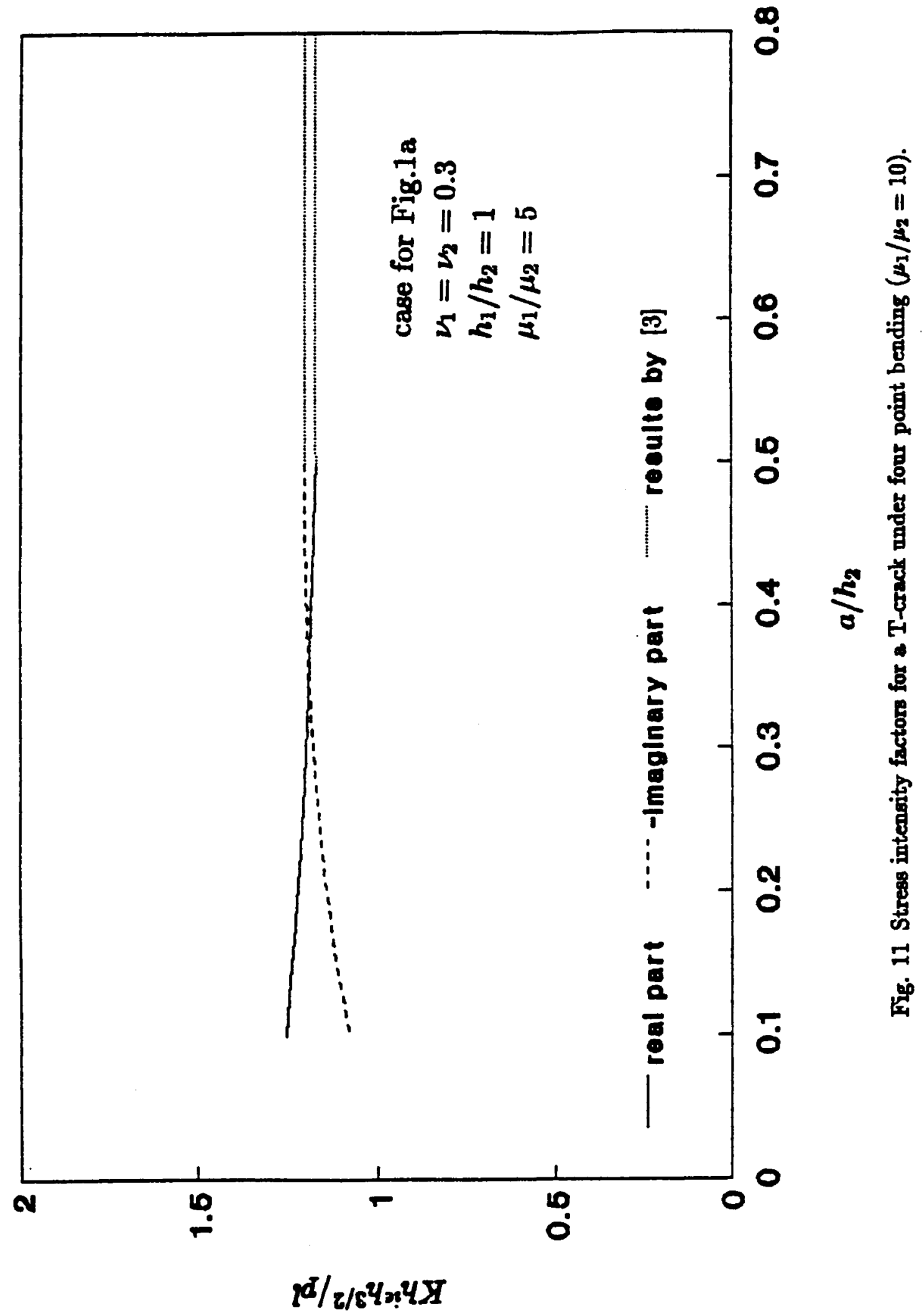




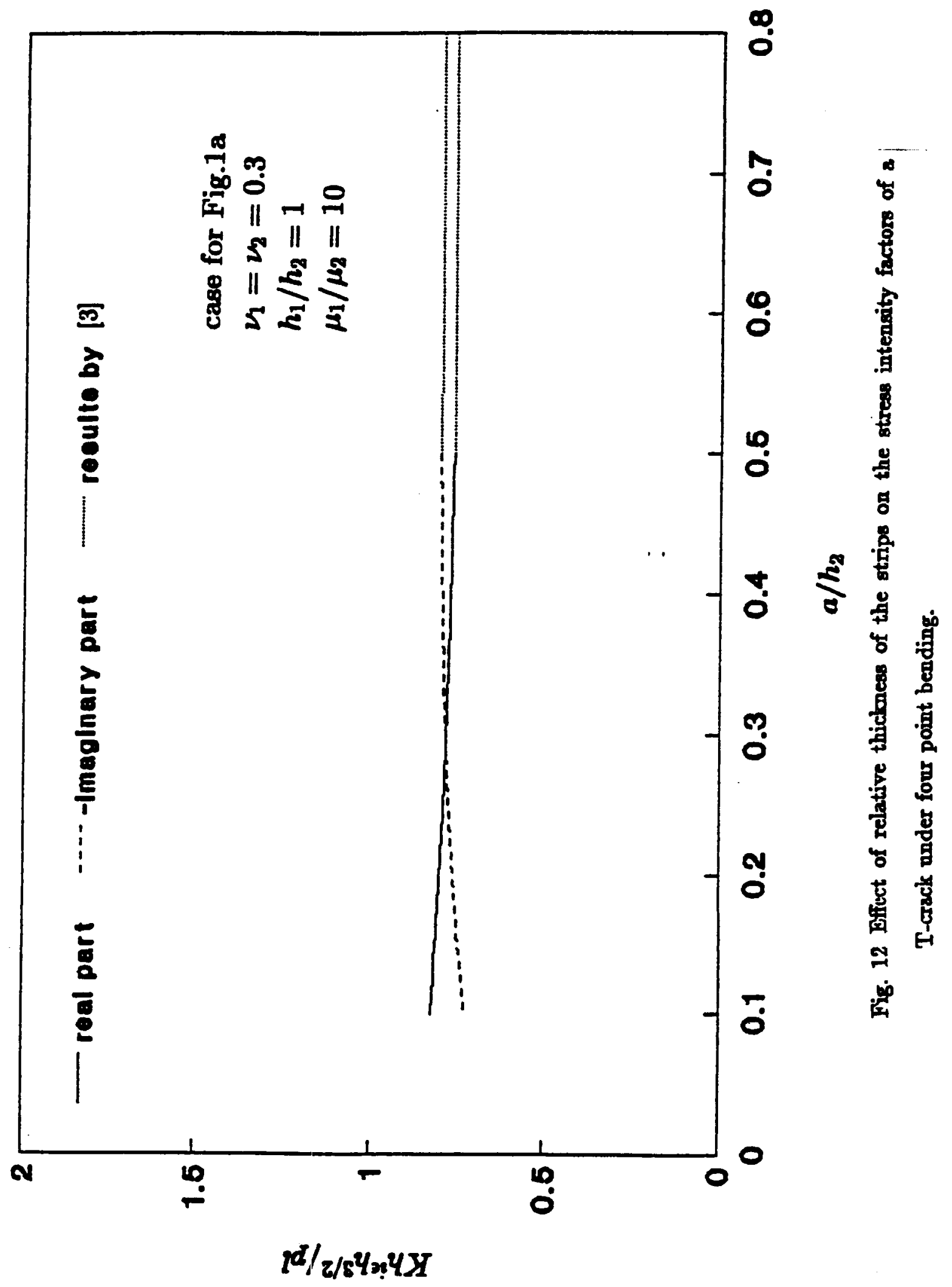




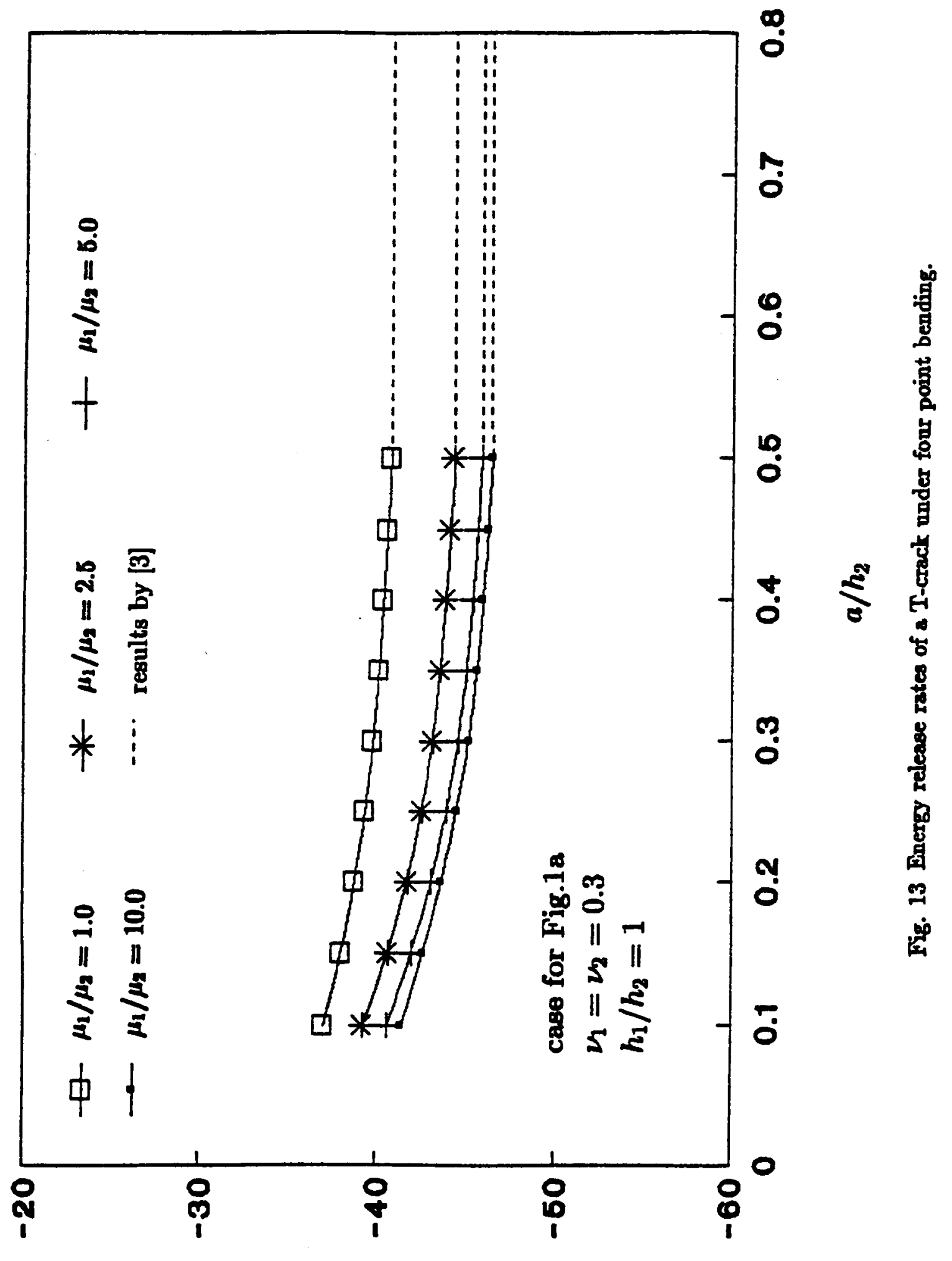

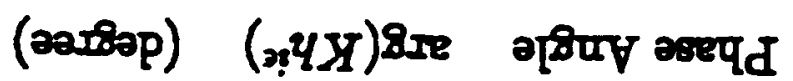




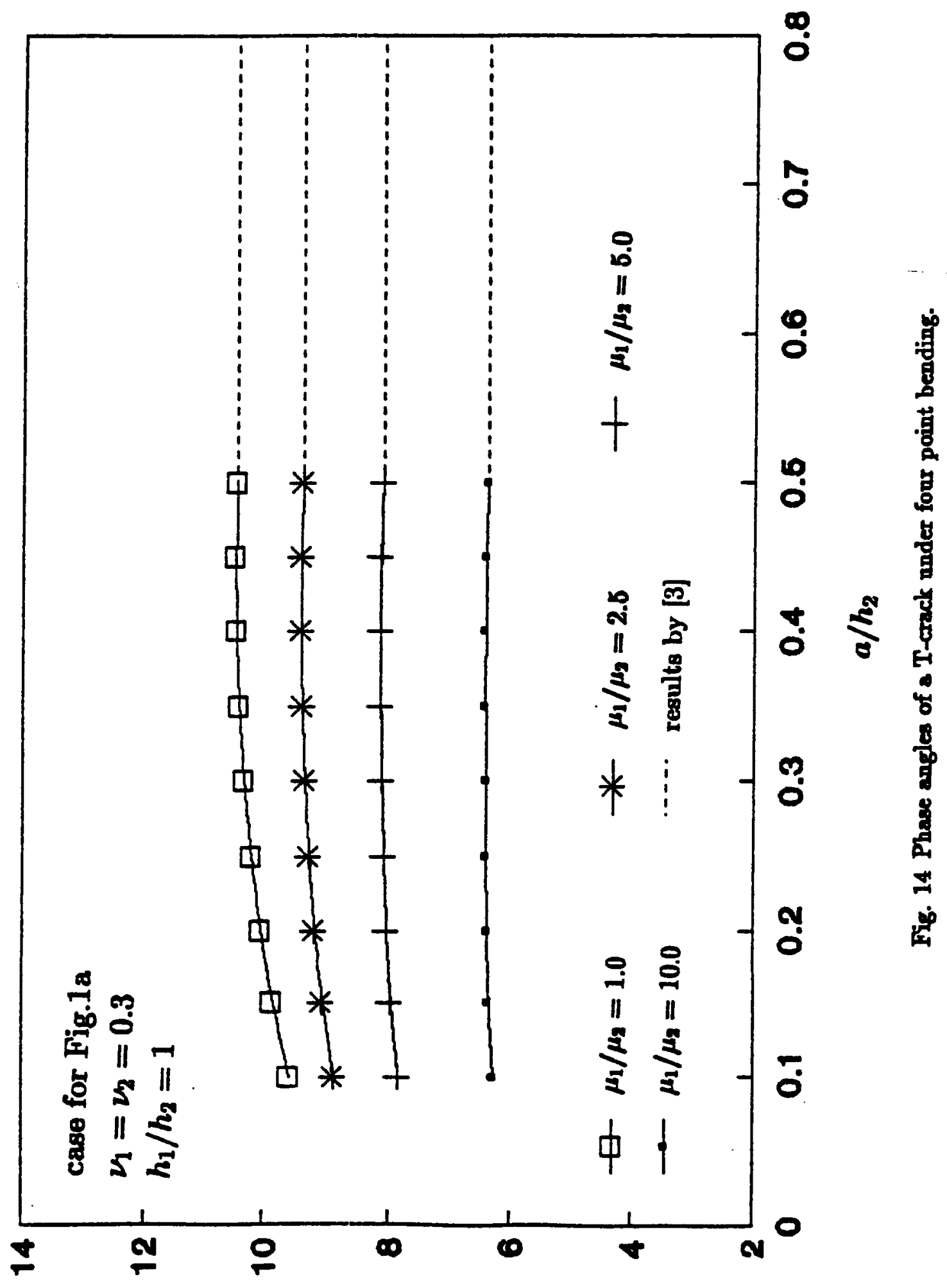

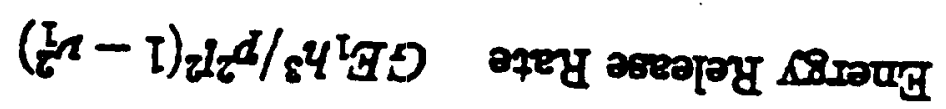




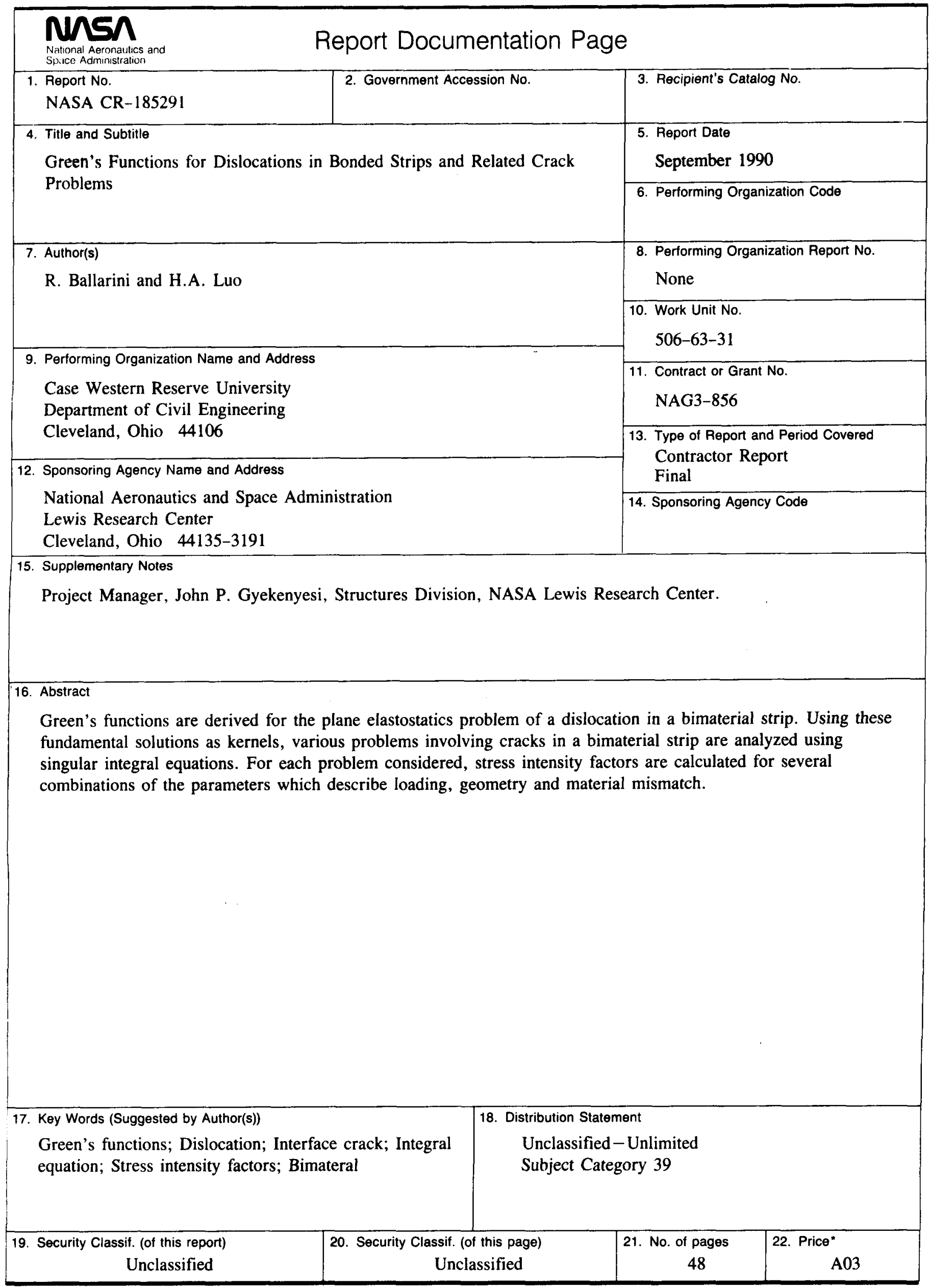

